\title{
UNIVERSAL INEQUALITIES FOR THE EIGENVALUES OF A POWER OF THE LAPLACE OPERATOR
}

\author{
SAÏD ILIAS AND OLA MAKHOUL
}

\begin{abstract}
In this paper, we obtain a new abstract formula relating eigenvalues of a self-adjoint operator to two families of symmetric and skew-symmetric operators and their commutators. This formula generalizes earlier ones obtained by Harrell, Stubbe, Hook, Ashbaugh, Hermi, Levitin and Parnovski. We also show how one can use this abstract formulation both for giving different and simpler proofs for all the known results obtained for the eigenvalues of a power of the Laplace operator (i.e. the Dirichlet Laplacian, the clamped plate problem for the bilaplacian and more generally for the polyharmonic problem on a bounded Euclidean domain) and to obtain new ones. In a last paragraph, we derive new bounds for eigenvalues of any power of the Kohn Laplacian on the Heisenberg group.
\end{abstract}

\section{INTRODUCTION}

Let $\Omega$ be a bounded domain of an n-dimensional Euclidean space $\mathbb{R}^{n}$ and consider the following eigenvalue problem for the polyharmonic operator :

$$
\left\{\begin{array}{l}
(-\Delta)^{l} u=\lambda u \text { in } \Omega \\
u=\frac{\partial u}{\partial \nu}=\cdots=\frac{\partial^{l-1} u}{\partial \nu^{l-1}}=0 \text { on } \partial \Omega,
\end{array}\right.
$$

where $\Delta$ is the Laplace operator and $\nu$ is the outward unit normal. It is known that this eigenvalue problem has a discrete spectrum,

$$
0<\lambda_{1}<\lambda_{2} \leq \cdots \leq \lambda_{k} \leq \ldots \rightarrow+\infty
$$

In this paper we will be interested in "Universal" (i.e. not depending on the domain) inequalities for the eigenvalues of such a polyharmonic problem and especially we will show how to derive them from a general abstract algebraic formula in the spirit of the work of Harrell, Stubbe, Ashbaugh and Hermi.

Let us begin by giving a short and non-exhaustive presentation of the known results in this field.

Date: 09 novembre 2009.

2000 Mathematics Subject Classification. 35P15;58C40.

Key words and phrases. eigenvalues, Laplacian, polyharmonic operator, biharmonic operator, clamped plate, Payne-Polya-Weinberger inequality, Hile-Protter inequality, Yang inequality, universal inequalities, commutators, Kohn Laplacian, Heisenberg group. 
The first result concerns the Dirichlet Laplacian (i.e. when $l=1$ ). In this case, Polya, Payne and Weinberger (henceforth PPW) proved in 1955 the following bound (see [26] for dimension 2 and [27] for all dimensions), for $k=1,2, \ldots$

$$
\lambda_{k+1}-\lambda_{k} \leq \frac{4}{n k} \sum_{i=1}^{k} \lambda_{i},
$$

This result was improved in 1980 by Hile and Protter [20] (henceforth HP) who showed that, for $k=1,2, \ldots$

$$
\frac{n k}{4} \leq \sum_{i=1}^{k} \frac{\lambda_{i}}{\lambda_{k+1}-\lambda_{i}}
$$

In 1991, H.C.Yang (see [29] and more recently [12]) proved

$$
\sum_{i=1}^{k}\left(\lambda_{k+1}-\lambda_{i}\right)^{2} \leq \frac{4}{n} \sum_{i=1}^{k} \lambda_{i}\left(\lambda_{k+1}-\lambda_{i}\right),
$$

which is, until now, the best improvement of the PPW inequality. From inequality (1.4), we can infer a weaker form

$$
\lambda_{k+1} \leq\left(1+\frac{4}{n}\right) \frac{1}{k}\left(\sum_{i=1}^{k} \lambda_{i}\right) .
$$

We shall refer to inequality (1.4) as Yang's first inequality (or simply Yang inequality) and to inequality (1.5) as Yang's second inequality.

The comparison of all these inequalities (see [2]) can be summarized in

$$
\text { Yang } 1 \Longrightarrow \text { Yang } 2 \Longrightarrow \mathrm{HP} \Longrightarrow \mathrm{PPW}
$$

When $l=2$, the eigenvalue problem (1.1) for the bilaplacian is the clamped plate problem. In the same paper as before [26], Polya, Payne and Weinberger proved the following analog of the formula (1.2)

$$
\lambda_{k+1}-\lambda_{k} \leq \frac{8(n+2)}{n^{2} k} \sum_{i=1}^{k} \lambda_{i} .
$$

And as was noticed by Ashbaugh (see [1] inequality (3.56)), there is a better inequality which was implicit in the PPW work,

$$
\lambda_{k+1}-\lambda_{k} \leq \frac{8(n+2)}{n^{2} k^{2}}\left(\sum_{i=1}^{k} \lambda_{i}^{\frac{1}{2}}\right)^{2} .
$$

In 1984, Hile and Yeh 21] extended the approach used for the Laplacian in [20] and proved the sharpest bound

$$
\frac{n^{2} k^{\frac{3}{2}}}{8(n+2)} \leq\left(\sum_{i=1}^{k} \frac{\lambda_{i}^{\frac{1}{2}}}{\lambda_{k+1}-\lambda_{i}}\right)\left(\sum_{i=1}^{k} \lambda_{i}\right)^{\frac{1}{2}} .
$$


Then in 1990, Hook [22], Chen and Qian [7] proved independently the following stronger inequality which was again implicit in the work of Hile and Yeh (see also [1, 8], 9] and [6])

$$
\frac{n^{2} k^{2}}{8(n+2)} \leq\left(\sum_{i=1}^{k} \frac{\lambda_{i}^{\frac{1}{2}}}{\lambda_{k+1}-\lambda_{i}}\right)\left(\sum_{i=1}^{k} \lambda_{i}^{\frac{1}{2}}\right) .
$$

Using Chebyshev inequality, Ashbaugh (see [1] inequality (3.60)) deduces from the preceding inequality (1.8), the following HP version which is weaker and more esthetically appealing,

$$
\frac{n^{2} k}{8(n+2)} \leq \sum_{i=1}^{k} \frac{\lambda_{i}}{\lambda_{k+1}-\lambda_{i}}
$$

Recently, Cheng and Yang [11] established the following Yang version

$$
\sum_{i=1}^{k}\left(\lambda_{k+1}-\lambda_{i}\right) \leq\left[\frac{8(n+2)}{n^{2}}\right]^{\frac{1}{2}} \sum_{i=1}^{k}\left[\lambda_{i}\left(\lambda_{k+1}-\lambda_{i}\right)\right]^{\frac{1}{2}} .
$$

For any $l$, the PPW inequality is given by

$$
\lambda_{k+1}-\lambda_{k} \leq \frac{4 l(2 l+n-2)}{n^{2} k^{2}}\left(\sum_{i=1}^{k} \lambda_{i}^{\frac{1}{l}}\right)\left(\sum_{i=1}^{k} \lambda_{i}^{\frac{l-1}{l}}\right) .
$$

Its HP improvement was proved independently by Hook [22] and Chen and Qian [7], it reads

$$
\frac{n^{2} k^{2}}{4 l(2 l+n-2)} \leq \sum_{i=1}^{k} \frac{\lambda_{i}^{\frac{1}{l}}}{\lambda_{k+1}-\lambda_{i}} \sum_{i=1}^{k} \lambda_{i}^{\frac{l-1}{l}} .
$$

As in the case $l=2$ (inequality (1.9)), this reduces to the weaker form

$$
\frac{n^{2} k}{4 l(2 l+n-2)} \leq \sum_{i=1}^{k} \frac{\lambda_{i}}{\lambda_{k+1}-\lambda_{i}}
$$

In 2007, Wu and Cao [28] generalized the inequality (1.10) of Cheng and Yang to the polyharmonic problem and obtained

$$
\begin{gathered}
\sum_{i=1}^{k}\left(\lambda_{k+1}-\lambda_{i}\right) \leq \\
\frac{1}{n}(4 l(n+2 l-2))^{\frac{1}{2}}\left(\sum_{i=1}^{k}\left(\lambda_{k+1}-\lambda_{i}\right)^{\frac{1}{2}} \lambda_{i}^{\frac{l-1}{l}}\right)^{\frac{1}{2}}\left(\sum_{i=1}^{k}\left(\lambda_{k+1}-\lambda_{i}\right)^{\frac{1}{2}} \lambda_{i}^{\frac{1}{l}}\right)^{\frac{1}{2}} .
\end{gathered}
$$

This inequality is sharper than inequality (1.11) (see [28]).

Very recently, Cheng, Ichikawa and Mametsuka [10] derived the following Yang type inequality for the polyharmonic operator (i.e. such that 
for $l=1$, we have the Yang inequality (1.4))

$$
\sum_{i=1}^{k}\left(\lambda_{k+1}-\lambda_{i}\right)^{2} \leq \frac{4 l(2 l+n-2)}{n^{2}} \sum_{i=1}^{k}\left(\lambda_{k+1}-\lambda_{i}\right) \lambda_{i} .
$$

All the classical proofs of these inequalities are based on tricky and careful choices of trial functions. For a more comprehensive and general approach, it is important to see if all these inequalities can be deduced using purely algebraic arguments involving eigenvalues and eigenfunctions of an abstract self-adjoint operator acting on a Hilbert space. In the case of the Laplacian (i.e. $l=1$ ), this was done by Harrell [14, 17], Harrell and Michel [16, 15], Harrell and Stubbe [19], and Ashbaugh and Hermi [4].

For the polyharmonic problem (i.e. general $l$ ), Hook [22] generalized the argument of Hile and Protter [20] in an abstract setting. Later, this abstract formulation of Hook was simplified and improved by Ashbaugh and Hermi [3]. In fact, they obtained the following inequality relating eigenvalues of a self-adjoint operator $A$, to two families of symmetric operators $B_{p}^{\prime} s$, skew-symmetric operators $T_{p}^{\prime} s, p=1, \ldots, n$ and their commutators (for a precise statement with detailed assumptions, see Theorem 2.2 of [3]),

$$
\frac{1}{4} \frac{\left(\sum_{i=1}^{k} \sum_{p=1}^{n}\left\langle\left[B_{p}, T_{p}\right] u_{i}, u_{i}\right\rangle\right)^{2}}{\sum_{i=1}^{k} \sum_{p=1}^{n}\left\langle\left[A, B_{p}\right] u_{i}, B_{p} u_{i}\right\rangle} \leq \sum_{i=1}^{k} \sum_{p=1}^{n} \frac{\left\langle T_{p} u_{i}, T_{p} u_{i}\right\rangle}{\lambda_{k+1}-\lambda_{i}} .
$$

But this abstract inequality, as was observed by Ashbaugh and Hermi in the end of the third paragraph of their article [3], could not recover more than the HP version of the universal inequalities (i.e. inequalities (1.11) and (1.12) ).

The main goal of the present paper is to prove the following abstract inequality (with the same assumptions as those for the Ashbaugh-Hermi inequality (1.15) which generalizes (1.15) and fills this gap

$$
\begin{gathered}
\left(\sum_{i=1}^{k} \sum_{p=1}^{n} f\left(\lambda_{i}\right)\left\langle\left[T_{p}, B_{p}\right] u_{i}, u_{i}\right\rangle\right)^{2} \\
\leq 4\left(\sum_{i=1}^{k} \sum_{p=1}^{n} g\left(\lambda_{i}\right)\left\langle\left[A, B_{p}\right] u_{i}, B_{p} u_{i}\right\rangle\right)\left(\sum_{i=1}^{k} \sum_{p=1}^{n} \frac{\left(f\left(\lambda_{i}\right)\right)^{2}}{g\left(\lambda_{i}\right)\left(\lambda_{k+1}-\lambda_{i}\right)}\left\|T_{p} u_{i}\right\|^{2}\right),
\end{gathered}
$$

where $f$ and $g$ are two functions satisfying some functional conditions (see Definition (2.1)). The family of such couples of functions is large and particular choices for $f$ and $g$ give many of the known universal inequalities. For instance, in the case of the polyharmonic problem, if we take $f(x)=g(x)=\left(\lambda_{k+1}-x\right)^{2}$, then we obtain the Yang type inequality (1.14) proved by Cheng, Ichikawa and Mametsuka and when we take $f(x)=(g(x))^{2}=\left(\lambda_{k+1}-x\right)$, we obtain the Wu-Cao inequality 
(1.13).

On the other hand, we observe that by taking $T_{p}=\left[A, B_{p}\right]$, we obtain the following new formula (see Corollary 2.1), where only one family of symmetric operators $B_{p}$ is needed

$$
\begin{gathered}
{\left[\sum_{i=1}^{k} \sum_{p=1}^{n} f\left(\lambda_{i}\right)\left\langle\left[A, B_{p}\right] u_{i}, B_{p} u_{i}\right\rangle\right]^{2}} \\
\leq\left[\sum_{i=1}^{k} \sum_{p=1}^{n} g\left(\lambda_{i}\right)\left\langle\left[A, B_{p}\right] u_{i}, B_{p} u_{i}\right\rangle\right]\left[\sum_{i=1}^{k} \sum_{p=1}^{n} \frac{\left(f\left(\lambda_{i}\right)\right)^{2}}{g\left(\lambda_{i}\right)\left(\lambda_{k+1}-\lambda_{i}\right)}\left\|\left[A, B_{p}\right] u_{i}\right\|^{2}\right] .
\end{gathered}
$$

Using this last inequality, with particular choices of $f$ and $g$ as before, one can recover many of the known universal inequalities for eigenvalues of Laplace or Schrödinger operators.

In the last section of this paper, we show how one can use the inequality (1.16) to derive new universal bounds, of Yang type, for eigenvalues of the Kohn Laplacian on the Heisenberg group, with any order. These bounds are stronger than the earlier bounds obtained by Niu and Zhang in 25 .

\section{The abstract formulation}

Before stating the main result of this section, we introduce a special family of couples of functions which will play an important role in our formulation.

Definition 2.1. Let $\lambda>0$. A couple $(f, g)$ of functions defined on ]0, $\lambda\left[\right.$ belongs to $\Im_{\lambda}$ provided that

1. $f$ and $g$ are positive,

2. $f$ and $g$ satisfy the following condition, for any $x, y \in] 0, \lambda[$ such that $x \neq y$,

$$
\left(\frac{f(x)-f(y)}{x-y}\right)^{2}+\left(\frac{(f(x))^{2}}{g(x)(\lambda-x)}+\frac{(f(y))^{2}}{g(y)(\lambda-y)}\right)\left(\frac{g(x)-g(y)}{x-y}\right) \leq 0 .
$$

A direct consequence of our definition is that $g$ must be nonincreasing.

If we multiply $f$ and $g$ of $\Im_{\lambda}$ by positive constants the resulting functions are also in $\Im_{\lambda}$. In the case where $f$ and $g$ are differentiable, one can easily deduce from (2.1) the following necessary condition:

$$
\left[(\ln f(x))^{\prime}\right]^{2} \leq \frac{-2}{\lambda-x}(\ln g(x))^{\prime} .
$$


This last condition helps us to find many couples $(f, g)$ satisfying the conditions 1) and 2) above. Among them, we mention $\left\{\left(1,(\lambda-x)^{\alpha}\right) / \alpha \geq 0\right\}$, $\left\{\left((\lambda-x),(\lambda-x)^{\beta}\right) / \beta \geq \frac{1}{2}\right\},\left\{\left((\lambda-x)^{\delta},(\lambda-x)^{\delta}\right) / 0<\delta \leq 2\right\}$. and $\left\{\left((\lambda-x)^{\alpha},(\lambda-x)^{\beta}\right) / \alpha<0,1 \leq \beta\right.$, and $\left.\alpha^{2} \leq \beta\right\}$.

Let $\mathcal{H}$ be a complex Hilbert space with scalar product $\langle.,$.$\rangle and$ corresponding norm $\|$.$\| . For any two operators A$ and $B$, we denote by $[A, B]$ their commutator, defined by $[A, B]=A B-B A$.

Theorem 2.1. Let $A: \mathcal{D} \subset \mathcal{H} \longrightarrow \mathcal{H}$ be a self-adjoint operator defined on a dense domain $\mathcal{D}$, which is semibounded below and has a discrete spectrum $\lambda_{1} \leq \lambda_{2} \leq \lambda_{3} \ldots$. Let $\left\{T_{p}: \mathcal{D} \longrightarrow \mathcal{H}\right\}_{p=1}^{n}$ be a collection of skew-symmetric operators, and $\left\{B_{p}: T_{p}(\mathcal{D}) \longrightarrow \mathcal{H}\right\}_{p=1}^{n}$ be a collection of symmetric operators, leaving $\mathcal{D}$ invariant. We denote by $\left\{u_{i}\right\}_{i=1}^{\infty} a$ basis of orthonormal eigenvectors of $A, u_{i}$ corresponding to $\lambda_{i}$. Let $k \geq 1$ and assume that $\lambda_{k+1}>\lambda_{k}$. Then, for any $(f, g)$ in $\Im_{\lambda_{k+1}}$

$$
\begin{gathered}
\left(\sum_{i=1}^{k} \sum_{p=1}^{n} f\left(\lambda_{i}\right)\left\langle\left[T_{p}, B_{p}\right] u_{i}, u_{i}\right\rangle\right)^{2} \\
\leq 4\left(\sum_{i=1}^{k} \sum_{p=1}^{n} g\left(\lambda_{i}\right)\left\langle\left[A, B_{p}\right] u_{i}, B_{p} u_{i}\right\rangle\right)\left(\sum_{i=1}^{k} \sum_{p=1}^{n} \frac{\left(f\left(\lambda_{i}\right)\right)^{2}}{g\left(\lambda_{i}\right)\left(\lambda_{k+1}-\lambda_{i}\right)}\left\|T_{p} u_{i}\right\|^{2}\right) .
\end{gathered}
$$

Proof of Theorem 2.1. For each $i$, we consider the vectors $\phi_{i}^{p}$, given by

$$
\phi_{i}^{p}=B_{p} u_{i}-\sum_{j=1}^{k} a_{i j}^{p} u_{j}
$$

where $a_{i j}^{p}:=\left\langle B_{p} u_{i}, u_{j}\right\rangle, p=1, \ldots, n$. We have

$$
\left\langle\phi_{i}^{p}, u_{j}\right\rangle=0
$$

for all $j=1, \ldots, k$. Taking $\phi_{i}^{p}$ as a trial vector in the Rayleigh-Ritz ratio, we obtain

$$
\lambda_{k+1} \leq \frac{\left\langle A \phi_{i}^{p}, \phi_{i}^{p}\right\rangle}{\left\langle\phi_{i}^{p}, \phi_{i}^{p}\right\rangle}
$$


Since $B_{p}$ is symmetric, for all $p=1, \ldots, n$, we have $a_{i j}^{p}=\overline{a_{j i}^{p}}$. Moreover, using the orthogonality conditions (2.3), we obtain

$$
\begin{aligned}
\left\|\phi_{i}^{p}\right\|^{2} & =\left\langle\phi_{i}^{p}, B_{p} u_{i}-\sum_{j=1}^{k} a_{i j}^{p} u_{j}\right\rangle=\left\langle\phi_{i}^{p}, B_{p} u_{i}\right\rangle \\
& =\left\langle B_{p} u_{i}-\sum_{j=1}^{k} a_{i j}^{p} u_{j}, B_{p} u_{i}\right\rangle=\left\|B_{p} u_{i}\right\|^{2}-\sum_{j=1}^{k} a_{i j}^{p}\left\langle B_{p} u_{i}, u_{j}\right\rangle \\
& =\left\|B_{p} u_{i}\right\|^{2}-\sum_{j=1}^{k}\left|a_{i j}^{p}\right|^{2}
\end{aligned}
$$

and

$$
\begin{aligned}
\left\langle A \phi_{i}^{p}, \phi_{i}^{p}\right\rangle & =\left\langle A B_{p} u_{i}-\sum_{j=1}^{k} \lambda_{j} a_{i j}^{p} u_{j}, \phi_{i}^{p}\right\rangle \\
& =\left\langle A B_{p} u_{i}, \phi_{i}^{p}\right\rangle \\
& =\left\langle A B_{p} u_{i}, B_{p} u_{i}\right\rangle-\sum_{j=1}^{k} \overline{a_{i j}^{p}}\left\langle A B_{p} u_{i}, u_{j}\right\rangle \\
& =\left\langle\left[A, B_{p}\right] u_{i}, B_{p} u_{i}\right\rangle+\left\langle B_{p} A u_{i}, B_{p} u_{i}\right\rangle-\sum_{j=1}^{k} \lambda_{j}\left|a_{i j}^{p}\right|^{2} \\
& =\left\langle\left[A, B_{p}\right] u_{i}, B_{p} u_{i}\right\rangle+\lambda_{i}\left\|B_{p} u_{i}\right\|^{2}-\sum_{j=1}^{k} \lambda_{j}\left|a_{i j}^{p}\right|^{2} .
\end{aligned}
$$

Hence, inequality (2.4) reduces to

$$
\lambda_{k+1}\left\|\phi_{i}^{p}\right\|^{2} \leq\left\langle\left[A, B_{p}\right] u_{i}, B_{p} u_{i}\right\rangle+\lambda_{i}\left\|B_{p} u_{i}\right\|^{2}-\sum_{j=1}^{k} \lambda_{j}\left|a_{i j}^{p}\right|^{2} .
$$

On the other hand, we observe that, for $p=1, \cdots, n$,

$$
\begin{aligned}
-2\left\langle T_{p} u_{i}, \phi_{i}^{p}\right\rangle & =-2\left\langle T_{p} u_{i}, B_{p} u_{i}\right\rangle+2\left\langle T_{p} u_{i}, \sum_{j=1}^{k} a_{i j}^{p} u_{j}\right\rangle \\
& =2\left\langle u_{i}, T_{p} B_{p} u_{i}\right\rangle+2 \sum_{j=1}^{k} \overline{a_{i j}^{p}}\left\langle T_{p} u_{i}, u_{j}\right\rangle \\
& =2\left\langle u_{i}, T_{p} B_{p} u_{i}\right\rangle+2 \sum_{j=1}^{k} \overline{a_{i j}^{p}} c_{i j}^{p},
\end{aligned}
$$

where $c_{i j}^{p}=\left\langle T_{p} u_{i}, u_{j}\right\rangle$.

Note that, since $T_{p}$ is skew-symmetric, we have $c_{i j}^{p}=-\overline{c_{j i}^{p}}$ for $1 \leq p \leq n$. 
Therefore, using (2.3) and taking the real part of both sides of (2.8), we obtain, for any constant $\alpha_{i}>0$,

$$
\begin{aligned}
2 \operatorname{Re}\left\langle T_{p} B_{p} u_{i}, u_{i}\right\rangle+2 \sum_{j=1}^{k} \operatorname{Re}\left(\overline{a_{i j}^{p}} c_{i j}^{p}\right) & =-2 \operatorname{Re}\left\langle\phi_{i}^{p}, T_{p} u_{i}\right\rangle \\
& =2 \operatorname{Re}\left\langle\phi_{i}^{p},-T_{p} u_{i}+\sum_{j=1}^{k} c_{i j}^{p} u_{j}\right\rangle \\
& \leq \alpha_{i}\left\|\phi_{i}^{p}\right\|^{2}+\frac{1}{\alpha_{i}}\left\|-T_{p} u_{i}+\sum_{j=1}^{k} c_{i j}^{p} u_{j}\right\|^{2} \\
& =\alpha_{i}\left\|\phi_{i}^{p}\right\|^{2}+\frac{1}{\alpha_{i}}\left(\left\|T_{p} u_{i}\right\|^{2}-\sum_{j=1}^{k}\left|c_{i j}^{p}\right|^{2}\right) .
\end{aligned}
$$

Multiplying (2.9) by $f\left(\lambda_{i}\right)$ and taking $\alpha_{i}=\frac{\alpha\left(\lambda_{k+1}-\lambda_{i}\right) g\left(\lambda_{i}\right)}{f\left(\lambda_{i}\right)}$, where $\alpha$ is a positive constant and $i \leq k$, we infer from (2.7)

$$
\begin{aligned}
& 2 f\left(\lambda_{i}\right)\left(\operatorname{Re}\left\langle T_{p} B_{p} u_{i}, u_{i}\right\rangle+\sum_{j=1}^{k} \operatorname{Re}\left(\overline{a_{i j}^{p}} c_{i j}^{p}\right)\right) \\
& \leq \alpha_{i} f\left(\lambda_{i}\right)\left\|\phi_{i}^{p}\right\|^{2}+\frac{1}{\alpha_{i}} f\left(\lambda_{i}\right)\left(\left\|T_{p} u_{i}\right\|^{2}-\sum_{j=1}^{k}\left|c_{i j}^{p}\right|^{2}\right) \\
& =\alpha\left(\lambda_{k+1}-\lambda_{i}\right) g\left(\lambda_{i}\right)\left\|\phi_{i}^{p}\right\|^{2}+\frac{1}{\alpha} \frac{\left(f\left(\lambda_{i}\right)\right)^{2}}{\left(\lambda_{k+1}-\lambda_{i}\right) g\left(\lambda_{i}\right)}\left(\left\|T_{p} u_{i}\right\|^{2}-\sum_{j=1}^{k}\left|c_{i j}^{p}\right|^{2}\right) \\
& \leq \alpha g\left(\lambda_{i}\right)\left\langle\left[A, B_{p}\right] u_{i}, B_{p} u_{i}\right\rangle+\alpha g\left(\lambda_{i}\right) \lambda_{i}\left\|B_{p} u_{i}\right\|^{2}-\alpha g\left(\lambda_{i}\right) \sum_{j=1}^{k} \lambda_{j}\left|a_{i j}^{p}\right|^{2}-\alpha g\left(\lambda_{i}\right) \lambda_{i}\left\|\phi_{i}^{p}\right\|^{2} \\
& +\frac{1}{\alpha} \frac{\left(f\left(\lambda_{i}\right)\right)^{2}}{\left(\lambda_{k+1}-\lambda_{i}\right) g\left(\lambda_{i}\right)}\left(\left\|T_{p} u_{i}\right\|^{2}-\sum_{j=1}^{k}\left|c_{i j}^{p}\right|^{2}\right) .
\end{aligned}
$$


Summing over $i=1, \cdots, k$ and using (2.5), we get

$$
\begin{aligned}
& 2 \sum_{i=1}^{k} f\left(\lambda_{i}\right) \operatorname{Re}\left\langle T_{p} B_{p} u_{i}, u_{i}\right\rangle+2 \sum_{i, j=1}^{k} f\left(\lambda_{i}\right) \operatorname{Re}\left(\overline{a_{i j}^{p}} c_{i j}^{p}\right) \\
& \leq \alpha \sum_{i=1}^{k} g\left(\lambda_{i}\right)\left\langle\left[A, B_{p}\right] u_{i}, B_{p} u_{i}\right\rangle+\alpha \sum_{i=1}^{k} \lambda_{i} g\left(\lambda_{i}\right)\left\|B_{p} u_{i}\right\|^{2} \\
& -\alpha \sum_{i, j=1}^{k} \lambda_{j} g\left(\lambda_{i}\right)\left|a_{i j}^{p}\right|^{2}-\alpha \sum_{i=1}^{k} \lambda_{i} g\left(\lambda_{i}\right)\left\|\phi_{i}^{p}\right\|^{2} \\
& +\frac{1}{\alpha} \sum_{i=1}^{k} \frac{\left(f\left(\lambda_{i}\right)\right)^{2}}{\left(\lambda_{k+1}-\lambda_{i}\right) g\left(\lambda_{i}\right)}\left(\left\|T_{p} u_{i}\right\|^{2}-\sum_{j=1}^{k}\left|c_{i j}^{p}\right|^{2}\right) \\
& =\alpha \sum_{i=1}^{k} g\left(\lambda_{i}\right)\left\langle\left[A, B_{p}\right] u_{i}, B_{p} u_{i}\right\rangle+\alpha \sum_{i, j=1}^{k}\left(\lambda_{i}-\lambda_{j}\right) g\left(\lambda_{i}\right)\left|a_{i j}^{p}\right|^{2} \\
& +\frac{1}{\alpha} \sum_{i=1}^{k} \frac{\left(f\left(\lambda_{i}\right)\right)^{2}}{\left(\lambda_{k+1}-\lambda_{i}\right) g\left(\lambda_{i}\right)}\left(\left\|T_{p} u_{i}\right\|^{2}-\sum_{j=1}^{k}\left|c_{i j}^{p}\right|^{2}\right)
\end{aligned}
$$

Since $a_{i j}^{p}=\overline{a_{j i}^{p}}$ and $c_{i j}^{p}=-\overline{c_{j i}^{p}}$, we have

$$
2 \operatorname{Re}\left(\sum_{i, j=1}^{k} f\left(\lambda_{i}\right) \overline{a_{i j}^{p}} c_{i j}^{p}\right)=\operatorname{Re}\left(\sum_{i, j=1}^{k}\left(f\left(\lambda_{i}\right)-f\left(\lambda_{j}\right)\right) \overline{a_{i j}^{p}} c_{i j}^{p}\right)
$$

Using that $\left|c_{i j}^{p}\right|^{2}=\left|c_{j i}^{p}\right|^{2}$, we find

$$
-\frac{1}{\alpha} \sum_{i, j=1}^{k} \frac{\left(f\left(\lambda_{i}\right)\right)^{2}}{g\left(\lambda_{i}\right)\left(\lambda_{k+1}-\lambda_{i}\right)}\left|c_{i j}^{p}\right|^{2}=\frac{-1}{2 \alpha} \sum_{i, j=1}^{k}\left[\frac{\left(f\left(\lambda_{i}\right)\right)^{2}}{g\left(\lambda_{i}\right)\left(\lambda_{k+1}-\lambda_{i}\right)}+\frac{\left(f\left(\lambda_{j}\right)\right)^{2}}{g\left(\lambda_{j}\right)\left(\lambda_{k+1}-\lambda_{j}\right)}\right]\left|c_{i j}^{p}\right|^{2} .
$$

Moreover,

$$
\alpha \sum_{i, j=1}^{k} g\left(\lambda_{i}\right)\left(\lambda_{i}-\lambda_{j}\right)\left|a_{i j}^{p}\right|^{2}=\frac{\alpha}{2} \sum_{i, j=1}^{k}\left(g\left(\lambda_{i}\right)-g\left(\lambda_{j}\right)\right)\left(\lambda_{i}-\lambda_{j}\right)\left|a_{i j}^{p}\right|^{2} .
$$


Thus we infer from (2.11), (2.12), (2.13) and (2.14)

$$
\begin{aligned}
& 2 \sum_{i=1}^{k} f\left(\lambda_{i}\right) \operatorname{Re}\left\langle T_{p} B_{p} u_{i}, u_{i}\right\rangle+\sum_{i, j=1}^{k}\left(f\left(\lambda_{i}\right)-f\left(\lambda_{j}\right)\right) \operatorname{Re}\left(\overline{a_{i j}^{p}} c_{i j}^{p}\right) \\
& \leq \alpha \sum_{i=1}^{k} g\left(\lambda_{i}\right)\left\langle\left[A, B_{p}\right] u_{i}, B_{p} u_{i}\right\rangle+\frac{1}{\alpha} \sum_{i=1}^{k} \frac{\left(f\left(\lambda_{i}\right)\right)^{2}}{g\left(\lambda_{i}\right)\left(\lambda_{k+1}-\lambda_{i}\right)}\left\|T_{p} u_{i}\right\|^{2} \\
& +\frac{\alpha}{2} \sum_{i, j=1}^{k}\left(g\left(\lambda_{i}\right)-g\left(\lambda_{j}\right)\right)\left(\lambda_{i}-\lambda_{j}\right)\left|a_{i j}^{p}\right|^{2} \\
& -\frac{1}{2 \alpha} \sum_{i, j=1}^{k}\left[\frac{\left(f\left(\lambda_{i}\right)\right)^{2}}{g\left(\lambda_{i}\right)\left(\lambda_{k+1}-\lambda_{i}\right)}+\frac{\left(f\left(\lambda_{j}\right)\right)^{2}}{g\left(\lambda_{j}\right)\left(\lambda_{k+1}-\lambda_{j}\right)}\right]\left|c_{i j}^{p}\right|^{2} .
\end{aligned}
$$

But

$$
\begin{gathered}
\sum_{i, j=1}^{k}\left(f\left(\lambda_{j}\right)-f\left(\lambda_{i}\right)\right) \operatorname{Re}\left(\overline{a_{i j}^{p}} c_{i j}\right) \leq \frac{\alpha}{2} \sum_{i, j=1}^{k}\left(g\left(\lambda_{j}\right)-g\left(\lambda_{i}\right)\right)\left(\lambda_{i}-\lambda_{j}\right)\left|a_{i j}^{p}\right|^{2} \\
+\frac{1}{2 \alpha} \sum_{i, j=1}^{k} \frac{\left(f\left(\lambda_{j}\right)-f\left(\lambda_{i}\right)\right)^{2}}{\left(\lambda_{i}-\lambda_{j}\right)^{2}} \frac{\lambda_{i}-\lambda_{j}}{g\left(\lambda_{j}\right)-g\left(\lambda_{i}\right)}\left|c_{i j}^{p}\right|^{2} .
\end{gathered}
$$

From the condition (2.1) satisfied by $f$ and $g$, we infer

$$
\begin{aligned}
\sum_{i=1}^{k}\left(f\left(\lambda_{j}\right)-\right. & \left.f\left(\lambda_{i}\right)\right) \operatorname{Re}\left(\overline{a_{i j}^{p}} c_{i j}^{p}\right) \leq \frac{\alpha}{2} \sum_{i, j=1}^{k}\left(g\left(\lambda_{j}\right)-g\left(\lambda_{i}\right)\right)\left(\lambda_{i}-\lambda_{j}\right)\left|a_{i j}^{p}\right|^{2} \\
& +\frac{1}{2 \alpha} \sum_{i, j=1}^{k}\left(\frac{\left(f\left(\lambda_{i}\right)\right)^{2}}{g\left(\lambda_{i}\right)\left(\lambda_{k+1}-\lambda_{i}\right)}+\frac{\left(f\left(\lambda_{j}\right)\right)^{2}}{g\left(\lambda_{j}\right)\left(\lambda_{k+1}-\lambda_{j}\right)}\right)\left|c_{i j}^{p}\right|^{2} .
\end{aligned}
$$

Hence, taking sum on $p$, from 1 to $n$, in (2.15), we find

$$
\begin{gathered}
2 \sum_{i=1}^{k} \sum_{p=1}^{n} f\left(\lambda_{i}\right) \operatorname{Re}\left\langle T_{p} B_{p} u_{i}, u_{i}\right\rangle \\
\leq \alpha \sum_{i=1}^{k} \sum_{p=1}^{n} g\left(\lambda_{i}\right)\left\langle\left[A, B_{p}\right] u_{i}, B_{p} u_{i}\right\rangle+\frac{1}{\alpha} \sum_{i=1}^{k} \sum_{p=1}^{n} \frac{\left(f\left(\lambda_{i}\right)\right)^{2}}{\left(\lambda_{k+1}-\lambda_{i}\right) g\left(\lambda_{i}\right)}\left\|T_{p} u_{i}\right\|^{2} .
\end{gathered}
$$

Since $B_{p}$ is symmetric and $T_{p}$ is skew-symmetric, we have for all $p \leq n$,

$$
\begin{aligned}
2 \operatorname{Re}\left\langle T_{p} B_{p} u_{i}, u_{i}\right\rangle & =\left\langle T_{p} B_{p} u_{i}, u_{i}\right\rangle+\overline{\left\langle T_{p} B_{p} u_{i}, u_{i}\right\rangle} \\
& =\left\langle T_{p} B_{p} u_{i}, u_{i}\right\rangle-\overline{\left\langle u_{i}, B_{p} T_{p} u_{i}\right\rangle} \\
& =\left\langle\left[T_{p}, B_{p}\right] u_{i}, u_{i}\right\rangle
\end{aligned}
$$


and inequality (2.18) becomes

$$
\begin{gathered}
\sum_{i=1}^{k} \sum_{p=1}^{n} f\left(\lambda_{i}\right)\left\langle\left[T_{p}, B_{p}\right] u_{i}, u_{i}\right\rangle \\
\leq \alpha \sum_{i=1}^{k} \sum_{p=1}^{n} g\left(\lambda_{i}\right)\left\langle\left[A, B_{p}\right] u_{i}, B_{p} u_{i}\right\rangle+\frac{1}{\alpha} \sum_{i=1}^{k} \sum_{p=1}^{n} \frac{\left(f\left(\lambda_{i}\right)\right)^{2}}{\left(\lambda_{k+1}-\lambda_{i}\right) g\left(\lambda_{i}\right)}\left\|T_{p} u_{i}\right\|^{2},
\end{gathered}
$$

or equivalently

$$
\begin{gathered}
\alpha^{2} \sum_{i=1}^{k} \sum_{p=1}^{n} g\left(\lambda_{i}\right)\left\langle\left[A, B_{p}\right] u_{i}, B_{p} u_{i}\right\rangle \\
-\alpha \sum_{i=1}^{k} \sum_{p=1}^{n} f\left(\lambda_{i}\right)\left\langle\left[T_{p}, B_{p}\right] u_{i}, u_{i}\right\rangle+\sum_{i=1}^{k} \sum_{p=1}^{n} \frac{\left(f\left(\lambda_{i}\right)\right)^{2}}{\left(\lambda_{k+1}-\lambda_{i}\right) g\left(\lambda_{i}\right)}\left\|T_{p} u_{i}\right\|^{2} \geq 0 .
\end{gathered}
$$

To prove inequality (2.2), it suffices to show that

$$
\sum_{i=1}^{k} \sum_{p=1}^{n} g\left(\lambda_{i}\right)\left\langle\left[A, B_{p}\right] u_{i}, B_{p} u_{i}\right\rangle \geq 0 .
$$

In fact, if this is the case, the discriminant of the quadratic polynomial (2.20) must be nonpositive, i.e.

$$
\begin{gathered}
\left(\sum_{i=1}^{k} \sum_{p=1}^{n} f\left(\lambda_{i}\right)\left\langle\left[T_{p}, B_{p}\right] u_{i}, u_{i}\right\rangle\right)^{2} \\
-4\left(\sum_{i=1}^{k} \sum_{p=1}^{n} g\left(\lambda_{i}\right)\left\langle\left[A, B_{p}\right] u_{i}, B_{p} u_{i}\right\rangle\right)\left(\sum_{i=1}^{k} \sum_{p=1}^{n} \frac{\left(f\left(\lambda_{i}\right)\right)^{2}}{\left(\lambda_{k+1}-\lambda_{i}\right) g\left(\lambda_{i}\right)}\left\|T_{p} u_{i}\right\|^{2}\right) \leq 0 .
\end{gathered}
$$

which yields the theorem. We note that if we replace $T_{p}$ by $-T_{p}$, inequality (2.20) holds. Thus we can deduce that it holds for all real $\alpha$ and not only $\alpha>0$ proving that the coefficient of the quadratic term, i.e. $\sum_{i=1}^{k} \sum_{p=1}^{n} g\left(\lambda_{i}\right)\left\langle\left[A, B_{p}\right] u_{i}, B_{p} u_{i}\right\rangle$, is nonnegative. If it is equal to zero, then $\sum_{i=1}^{k} \sum_{p=1}^{n} f\left(\lambda_{i}\right)\left\langle\left[T_{p}, B_{p}\right] u_{i}, u_{i}\right\rangle$ is also equal to 0 and the theorem trivially holds.

Remark 2.1. - In the definition of $\Im_{\lambda}$, the functions $f$ and $g$ can be defined only on a discrete set of eigenvalues.

- One can formulate Theorem [2.1 as in [19] for $z \in] \lambda_{k}, \lambda_{k+1}$ ] (it suffices to replace, in the hypothesis and in the inequality, $\lambda_{k+1}$ by $z$ ). 
- The result of Theorem [2.1 can also be stated, as in [19] or [18], in the general situation where the spectrum of $A$ is not purely discrete and its point spectrum is nonempty.

- Taking $f=g=1$ in (2.2), we obtain inequality (1.15) of Ashbaugh and Hermi.

If the operators $T_{p}$ are chosen such that $T_{p}=\left[A, B_{p}\right]$, then $\left[T_{p}, B_{p}\right]=$ $\left[\left[A, B_{p}\right], B_{p}\right]$. Applying Theorem 2.1 in this context and using the obvious identity $\left\langle\left[A, B_{p}\right] u_{i}, B_{p} u_{i}\right\rangle=-\frac{1}{2}\left\langle\left[\left[A, B_{p}\right], B_{p}\right] u_{i}, u_{i}\right\rangle$, we obtain

Corollary 2.1. Let $A: \mathcal{D} \subset \mathcal{H} \longrightarrow \mathcal{H}$ be a self-adjoint operator defined on a dense domain $\mathcal{D}$, which is semibounded below and has a discrete spectrum $\lambda_{1} \leq \lambda_{2} \leq \lambda_{3} \ldots$ Let $\left\{B_{p}: A(\mathcal{D}) \longrightarrow \mathcal{H}\right\}_{p=1}^{n}$ be a collection of symmetric operators, leaving $\mathcal{D}$ invariant. We denote by $\left\{u_{i}\right\}_{i=1}^{\infty}$ a basis of orthonormal eigenvectors of $A, u_{i}$ corresponding to $\lambda_{i}$. If for $k \geq 1$ we have $\lambda_{k+1}>\lambda_{k}$, then for any $(f, g) \in \Im_{k}$,

$$
\begin{gathered}
{\left[\sum_{i=1}^{k} \sum_{p=1}^{n} f\left(\lambda_{i}\right)\left\langle\left[A, B_{p}\right] u_{i}, B_{p} u_{i}\right\rangle\right]^{2}} \\
\leq\left[\sum_{i=1}^{k} \sum_{p=1}^{n} g\left(\lambda_{i}\right)\left\langle\left[A, B_{p}\right] u_{i}, B_{p} u_{i}\right\rangle\right]\left[\sum_{i=1}^{k} \sum_{p=1}^{n} \frac{\left(f\left(\lambda_{i}\right)\right)^{2}}{g\left(\lambda_{i}\right)\left(\lambda_{k+1}-\lambda_{i}\right)}\left\|\left[A, B_{p}\right] u_{i}\right\|^{2}\right] .
\end{gathered}
$$

Remark 2.2. $\quad$ - As for Theorem 2.1, Corollary 2.1 can be stated in the general case where the spectrum of $A$ is not totally discrete.

- For $f(x)=g(x)=\left(\lambda_{k+1}-x\right)^{2}$, inequality (2.23) becomes the abstract inequality which gives the Yang type inequalities for Laplacians and Schrödinger operators (see [4], [13], [19] and [24]).

- For $f(x)=g(x)=\left(\lambda_{k+1}-x\right)^{\alpha}$, with $\alpha \leq 2$, we recover a Harrell and Stubbe inequality (19], [5])).

- We can easily deduce from the inequality (2.23) new universal inequalities in many different geometric situations (Dirichlet Laplacian on domains of Submanifolds of Euclidean (or symmetric) spaces as in [13], Hodge de Rham Laplacian or the square of a Dirac operator, and more generally a Laplacian acting on sections of a Riemannian vector bundle on a submanifold of a Euclidean (or symmetric) space).

\section{Application to the polyharmonic operators}

In this section, using Theorem 2.1, we will show how to derive universal inequalities for the eigenvalues of a polyharmonic problem. For a power of the Laplacian and with a particular choice of $f$ and $g$, one can derive inequality (1.13) and inequality (1.14). 
In fact, throughout this section we assume that $A=Q^{l}$, such that $Q$ is a symmetric self-adjoint operator given by

$$
Q=-\sum_{p=1}^{n} T_{p}^{2}
$$

where $T_{p}$ are skew-symmetric operators for $p=1, \ldots, n$, with $\left[Q, T_{p}\right]=0$ and $\left[T_{m}, B_{p}\right]=\delta_{m p}$.

First we need to calculate the following expressions

$$
\sum_{p=1}^{n}\left\langle\left[A, B_{p}\right] u_{i}, B_{p} u_{i}\right\rangle
$$

and

$$
\sum_{p=1}^{n}\left\langle T_{p} u_{i}, T_{p} u_{i}\right\rangle=\left\langle Q u_{i}, u_{i}\right\rangle .
$$

For this purpose the following two results of Hook (see proposition 3 in [22] and Theorem 1 in [23]) will be useful.

The first one is

Lemma 3.1. Under the circumstances stated above, we have

$$
\left[A, B_{p}\right]=\left[Q^{l}, B_{p}\right]=-2 l Q^{l-1} T_{p}
$$

and

$$
\sum_{p=1}^{n}\left[B_{p},\left[A, B_{p}\right]\right]=2 l(2 l+n-2) Q^{l-1} .
$$

And the second one is the following

Theorem 3.1. Let $V$ be a real or complex inner product space with inner product $\langle.,$.$\rangle . Let D$ be a linear submanifold of $V$ and let $Q$ : $D \longrightarrow V$ be a linear operator in $V$. Suppose $l$ is a positive integer and $u$ is a fixed vector such that for all $0 \leq r \leq q \leq l$,

$$
\left|\left\langle Q^{q} u, u\right\rangle\right|=\left|\left\langle Q^{q-r} u, Q^{r} u\right\rangle\right| .
$$

Then, for all integers $0 \leq r \leq q \leq l$, when $q$ is even, we have

$$
\left|\left\langle Q^{r} u, u\right\rangle\right| \leq\left|\left\langle Q^{q} u, u\right\rangle\right|^{r / q}\langle u, u\rangle^{1-r / q} .
$$

This inequality is satisfied for $q$ odd and $0 \leq r \leq q \leq l$, if in addition to the above, there is a family of operators $\left\{T_{p}\right\}_{p=1}^{n}$ such that

$$
\left|\left\langle Q^{q} u, u\right\rangle\right|=\left|\sum_{p=1}^{n}\left\langle T_{p} Q^{q-r} u, T_{p} Q^{r-1} u\right\rangle\right|
$$

holds for all $0 \leq r \leq q \leq l$. 
Applying Lemma 3.1, we obtain

$$
\begin{aligned}
\sum_{p=1}^{n}\left\langle\left[A, B_{p}\right] u_{i}, B_{p} u_{i}\right\rangle & =\frac{1}{2} \sum_{p=1}^{n}\left\langle\left[B_{p},\left[A, B_{p}\right]\right] u_{i}, u_{i}\right\rangle \\
& =l(2 l+n-2)\left\langle Q^{l-1} u_{i}, u_{i}\right\rangle .
\end{aligned}
$$

Therefore, if $l$ is odd, then we have

$$
\sum_{p=1}^{n}\left\langle\left[A, B_{p}\right] u_{i}, B_{p} u_{i}\right\rangle=l(2 l+n-2)\left\|Q^{\frac{l-1}{2}} u_{i}\right\|^{2}
$$

and if $l$ is even, then

$$
\sum_{p=1}^{n}\left\langle\left[A, B_{p}\right] u_{i}, B_{p} u_{i}\right\rangle=l(2 l+n-2) \sum_{p=1}^{n}\left\|T_{p} Q^{\frac{l-2}{2}} u_{i}\right\|^{2}
$$

The conditions of Theorem 3.1 are satisfied by our operator $Q$. So inequality (3.3) is valid for all $0 \leq r \leq q \leq l$ without parity condition on $q$. Applying this inequality (3.3) with $r=l-1$ and $q=l$, we obtain

$$
\begin{aligned}
\sum_{p=1}^{n}\left\langle\left[A, B_{p}\right] u_{i}, B_{p} u_{i}\right\rangle & =l(2 l+n-2)\left\langle Q^{l-1} u_{i}, u_{i}\right\rangle \\
& \leq l(2 l+n-2)\left\langle Q^{l} u_{i}, u_{i}\right\rangle^{\frac{l-1}{l}}\left\langle u_{i}, u_{i}\right\rangle^{1-\frac{l-1}{l}} \\
& =l(2 l+n-2) \lambda_{i}^{\frac{l-1}{l}}
\end{aligned}
$$

and with $r=1$ and $q=l$, we obtain

$$
\sum_{p=1}^{n}\left\|T_{p} u_{i}\right\|^{2}=\left\langle Q u_{i}, u_{i}\right\rangle \leq\left\langle Q^{l} u_{i}, u_{i}\right\rangle^{\frac{1}{l}}\left\langle u_{i}, u_{i}\right\rangle^{1-\frac{1}{l}} \leq \lambda_{i}^{\frac{1}{l}} .
$$

Since $\left[T_{p}, B_{p}\right]=1$, one gets

$$
\left\langle\left[T_{p}, B_{p}\right] u_{i}, u_{i}\right\rangle=1 \text {. }
$$

Then using inequalities (3.4), (3.5) and (3.6) together with inequality (2.2), we obtain

$n^{2}\left[\sum_{i=1}^{k} f\left(\lambda_{i}\right)\right]^{2} \leq 4 l(2 l+n-2)\left(\sum_{i=1}^{k} g\left(\lambda_{i}\right) \lambda_{i}^{\frac{l-1}{l}}\right)\left(\sum_{i=1}^{k} \frac{\left(f\left(\lambda_{i}\right)\right)^{2}}{g\left(\lambda_{i}\right)\left(\lambda_{k+1}-\lambda_{i}\right)} \lambda_{i}^{\frac{1}{l}}\right)$

or equivalently

$\sum_{i=1}^{k} f\left(\lambda_{i}\right) \leq \frac{2}{n} \sqrt{l(2 l+n-2)}\left(\sum_{i=1}^{k} g\left(\lambda_{i}\right) \lambda_{i}^{\frac{l-1}{l}}\right)^{\frac{1}{2}}\left(\sum_{i=1}^{k} \frac{\left(f\left(\lambda_{i}\right)\right)^{2}}{g\left(\lambda_{i}\right)\left(\lambda_{k+1}-\lambda_{i}\right)} \lambda_{i}^{\frac{1}{l}}\right)^{\frac{1}{2}}$.

Now the operators $A=(-\Delta)^{l}, Q=-\Delta, B_{p}=x_{p}, p=1, \ldots, n$, where $x_{1}, \ldots, x_{n}$ are Euclidean coordinates, and $T_{p}=\frac{\partial}{\partial x_{p}}$ fit the setup of this 
section. Thus, taking $f(x)=(g(x))^{2}=\left(\lambda_{k+1}-x\right)$, we can obtain inequality (1.13) of $\mathrm{Wu}$ and Cao.

Remark 3.1. For the special case $l=2$ (i.e the clamped plate problem) and the same values of $f$ and $g$ as above, we obtain inequality (1.10) of Cheng and Yang. We observe that this inequality can also be obtained easily by a simple calculation from our inequality (2.2). In fact, taking $A=\Delta^{2}, B_{p}=x_{p}, p=1, \ldots, n$ and $T_{p}=\frac{\partial}{\partial x_{p}}$, we first observe that $\left[T_{p}, B_{p}\right]=1$. Hence, we have

$$
\sum_{p=1}^{n}\left\langle\left[T_{p}, B_{p}\right] u_{i}, u_{i}\right\rangle=n,
$$

moreover

$$
\left[A, B_{p}\right] u_{i}=\left[\Delta^{2}, x_{p}\right] u_{i}=4 \frac{\partial}{\partial x_{p}} \Delta u_{i}
$$

Then

$$
\begin{aligned}
{\left[B_{p},\left[A, B_{p}\right]\right] u_{i} } & =4\left[x_{p}, \frac{\partial}{\partial x_{p}} \Delta\right] u_{i} \\
& =-4\left(\Delta+2\left(\frac{\partial}{\partial x_{p}}\right)^{2}\right) u_{i} .
\end{aligned}
$$

It follows that

$$
\begin{aligned}
\sum_{p=1}^{n}\left\langle\left[A, B_{p}\right] u_{i}, B_{p} u_{i}\right\rangle & =\frac{1}{2} \sum_{p=1}^{n}\left\langle\left[B_{p},\left[A, B_{p}\right]\right] u_{i}, u_{i}\right\rangle \\
& =2(n+2)\left\langle-\Delta u_{i}, u_{i}\right\rangle \\
& \leq 2(n+2)\left(\left\|\Delta u_{i}\right\|^{2}\left\|u_{i}\right\|^{2}\right)^{\frac{1}{2}} \\
& =2(n+2) \lambda_{i}^{\frac{1}{2}} .
\end{aligned}
$$

Now

$$
\sum_{p=1}^{n}\left\langle T_{p} u_{i}, T_{p} u_{i}\right\rangle=\left\langle-\Delta u_{i}, u_{i}\right\rangle \leq \lambda_{i}^{\frac{1}{2}},
$$

where we used the Cauchy-Schwarz inequality to derive (3.9) and (3.11). Substituting (3.8), (3.10) and (3.11) into (2.2) and taking $f(x)=$ $(g(x))^{2}=\left(\lambda_{k+1}-x\right)$, we obtain inequality (1.10).

On the other hand, if we take $f(x)=g(x)=\left(\lambda_{k+1}-x\right)^{2}$, in (3.7), we get the following inequality obtained in [10] (see inequality (2.27) 
therein)

$$
\begin{gathered}
{\left[\sum_{i=1}^{k}\left(\lambda_{k+1}-\lambda_{i}\right)^{2}\right]^{2}} \\
\leq \frac{4 l(2 l+n-2)}{n^{2}}\left(\sum_{i=1}^{k}\left(\lambda_{k+1}-\lambda_{i}\right)^{2} \lambda_{i}^{\frac{l-1}{l}}\right)\left(\sum_{i=1}^{k}\left(\lambda_{k+1}-\lambda_{i}\right) \lambda_{i}^{\frac{1}{l}}\right) .
\end{gathered}
$$

Using the following variant of Chebyshev inequality (see Lemma 1 in [10]), one can deduce a generalized Yang inequality

Lemma 3.2. Let $A_{i}, B_{i}$ and $C_{i}, i=1, \ldots, k$, verify $A_{1} \geq A_{2} \geq \ldots \geq$ $A_{k} \geq 0,0 \leq B_{1} \leq B_{2} \leq \ldots \leq B_{k}$ and $0 \leq C_{1} \leq C_{2} \leq \ldots \leq C_{k}$, respectively. Then, we have

$$
\sum_{i=1}^{k} A_{i}^{2} B_{i} \sum_{i=1}^{k} A_{i} C_{i} \leq \sum_{i=1}^{k} A_{i}^{2} \sum_{i=1}^{k} A_{i} B_{i} C_{i}
$$

In fact if we apply this Lemma to the right side of inequality (3.12), with $A_{i}=\lambda_{k+1}-\lambda_{i}, B_{i}=\lambda_{i}^{\frac{l-1}{l}}$ and $C_{i}=\lambda_{i}^{\frac{1}{l}}$, we obtain,

$$
\sum_{i=1}^{k}\left(\lambda_{k+1}-\lambda_{i}\right)^{2} \leq \frac{4 l(n+2 l-2)}{n^{2}} \sum_{i=1}^{k}\left(\lambda_{k+1}-\lambda_{i}\right) \lambda_{i} .
$$

which, in the case where $A=(-\Delta)^{l}, Q=-\Delta, B_{p}=x_{p}, p=1, \ldots, n$ and $T_{p}=\frac{\partial}{\partial x_{p}}$, gives us inequality (1.14) of Cheng, Ichikawa and Mametsuka (see inequality (1.11) in [10]).

Finally, we note that considering other choices of values for the couple $(f, g)$ lead to many new inequalities.

\section{Applications to the Kohn Laplacian on the Heisenberg GROUP}

In this section, we consider the $2 n+1$-dimensional Heisenberg group $\mathbb{H}^{n}$, which is the space $\mathbb{R}^{2 n+1}$ equipped with the non-commutative group law

$$
(x, y, t)\left(x^{\prime}, y^{\prime}, t^{\prime}\right)=\left(x+x^{\prime}, y+y^{\prime}, t+t^{\prime}+\frac{1}{2}\right)\left(\left\langle x^{\prime}, y\right\rangle_{\mathbb{R}^{n}}-\left\langle x, y^{\prime}\right\rangle_{\mathbb{R}^{n}}\right),
$$

where $x, x^{\prime}, y, y^{\prime} \in \mathbb{R}^{n}, t$ and $t^{\prime} \in \mathbb{R}$. We denote by $\mathcal{H}^{n}$ its Lie algebra, it has a basis formed by the following vector fields $T=\frac{\partial}{\partial t}$, $X_{p}=\frac{\partial}{\partial x_{p}}+\frac{y_{p}}{2} \frac{\partial}{\partial t}$ and $Y_{p}=\frac{\partial}{\partial y_{p}}-\frac{x_{p}}{2} \frac{\partial}{\partial t}$. We note that the only non-trivial 
commutators are $\left[Y_{p}, X_{q}\right]=T \delta_{p q}$. Let $\Delta_{\mathbb{H}^{n}}$ denote the real KohnLaplacian in the Heisenberg group $\mathbb{H}^{n}$. It is given by

$$
\begin{aligned}
\Delta_{\mathbb{H}^{n}} & =\sum_{p=1}^{n} X_{p}^{2}+Y_{p}^{2} \\
& =\Delta_{x y}^{\mathbb{R}^{2 n}}+\frac{1}{4}\left(|x|^{2}+|y|^{2}\right) \frac{\partial^{2}}{\partial t^{2}}+\frac{\partial}{\partial t} \sum_{p=1}^{n}\left(y_{p} \frac{\partial}{\partial x_{p}}-x_{p} \frac{\partial}{\partial y_{p}}\right) .
\end{aligned}
$$

We are concerned here with the following eigenvalue problem:

$$
\left\{\begin{array}{l}
\left(-\Delta_{\mathbb{H}^{n}}\right)^{l} u=\lambda u \text { in } \Omega, \\
u=\frac{\partial u}{\partial \nu}=\ldots=\frac{\partial^{l-1} u}{\partial \nu^{l-1}}=0 \text { on } \partial \Omega,
\end{array}\right.
$$

where $\Omega$ is a bounded domain in $\mathbb{H}^{n}$, with smooth boundary $\partial \Omega, \nu$ is the unit outward normal to $\partial \Omega$ and $l \geq 1$ is any positive integer. We denote by $L=-\Delta_{\mathbb{H}^{n}}$ and $\nabla_{\mathbb{H}^{n}}=\left(X_{1}, \ldots, X_{n}, Y_{1}, \ldots, Y_{n}\right)$.

We let

$$
0<\lambda_{1} \leq \lambda_{2} \leq \ldots \leq \lambda_{k} \leq \ldots \rightarrow+\infty
$$

denote the eigenvalues of problem (4.1) with corresponding eigenfunctions $u_{1}, u_{2}, \ldots, u_{k}, \ldots$ in $S_{0}^{l, 2}(\Omega)$. Here $S^{l, 2}(\Omega)$ is the Hilbert space of the functions $u$ in $L^{2}(\Omega)$ such that $X_{p} u, Y_{p} u, X_{p}^{2} u, Y_{p}^{2} u, \ldots, X_{p}^{l}(u)$, $Y_{p}^{l}(u) \in L^{2}(\Omega)$, and $S_{0}^{l, 2}$ denotes the closure of $C_{0}^{\infty}(\Omega)$ with respect to the Sobolev norm

$$
\|u\|_{S^{l, 2}}^{2}=\int_{\Omega}\left(\sum_{d=1}^{l}\left(\sum_{p=1}^{n}\left|X_{p}^{d} u\right|^{2}+\sum_{p=1}^{n}\left|Y_{p}^{d} u\right|^{2}\right)+|u|^{2}\right) d x d y d t .
$$

We orthonormalize the eigenfunctions $u_{i}$ so that; $\forall i, j \geq 1$,

$$
\left\langle u_{i}, u_{j}\right\rangle_{L^{2}}=\int_{\Omega} u_{i} u_{j} d x d y d t=\delta_{i j}
$$

In all this paragraph, our results can be stated in a general form using functions $f$ and $g \in \Im_{\lambda_{k+1}}$ as in the first part of this paper, but we limit ourselves to the case $f(x)=g(x)=\left(\lambda_{k+1}-x\right)^{2}$. This gives us new bounds of the Yang type for eigenvalues of problem (4.1) which improve earlier ones obtained by Niu and Zhang [25].

We also note that we must treat the three following cases independently: the case when $l=1$, the case when $l=2$ and the case when $l \geq 3$. This is essentially due to the difference of the calculations in these three cases.

4.1. The case when $\boldsymbol{l}=\mathbf{1}$. In this subsection, we are concerned with the case where $l=1$. The result we obtain is a result proved earlier by the first author, El Soufi and Harrell in [13] and for which we give here a different proof, more easily adapted to the other cases $l=2$ and $l \geq 3$. 
Theorem 4.1. For any $k \geq 1$

$$
\sum_{i=1}^{k}\left(\lambda_{k+1}-\lambda_{i}\right)^{2} \leq \frac{2}{n} \sum_{i=1}^{k}\left(\lambda_{k+1}-\lambda_{i}\right) \lambda_{i}
$$

Proof. We will prove this theorem by applying inequality (2.2) with $A=L=-\Delta_{\mathbb{H}^{n}}, B_{1}=x_{1}, \ldots, B_{n}=x_{n}, B_{n+1}=y_{1}, \ldots, B_{2 n}=y_{n}$, $T_{1}=X_{1}, \ldots, T_{n}=X_{n}, T_{n+1}=Y_{1}, \ldots, T_{2 n}=Y_{n}$ and $f(x)=g(x)=$ $\left(\lambda_{k+1}-x\right)^{2}$, namely,

$$
\begin{gathered}
{\left[\sum_{p=1}^{n} \sum_{i=1}^{k}\left(\lambda_{k+1}-\lambda_{i}\right)^{2}\left(\left\langle\left[X_{p}, x_{p}\right] u_{i}, u_{i}\right\rangle_{L^{2}}+\left\langle\left[Y_{p}, y_{p}\right] u_{i}, u_{i}\right\rangle_{L^{2}}\right)\right]^{2}} \\
\leq 4\left[\sum_{p=1}^{n} \sum_{i=1}^{k}\left(\lambda_{k+1}-\lambda_{i}\right)^{2}\left(\left\langle\left[L, x_{p}\right] u_{i}, x_{p} u_{i}\right\rangle_{L^{2}}+\left\langle\left[L, y_{p}\right] u_{i}, y_{p} u_{i}\right\rangle_{L^{2}}\right)\right] \times \\
{\left[\sum_{p=1}^{n} \sum_{i=1}^{k}\left(\lambda_{k+1}-\lambda_{i}\right)\left(\left\|X_{p} u_{i}\right\|_{L^{2}}^{2}+\left\|Y_{p} u_{i}\right\|_{L^{2}}^{2}\right)\right] .}
\end{gathered}
$$

By a straightforward calculation, we obtain $\left[L, x_{p}\right] u_{i}=-2 X_{p} u_{i}$ and $\left[L, y_{p}\right] u_{i}=-2 Y_{p} u_{i}$.

Hence

$$
\begin{aligned}
\left\langle\left[L, x_{p}\right] u_{i}, x_{p} u_{i}\right\rangle_{L^{2}} & =-2 \int_{\Omega} X_{p} u_{i} \cdot x_{p} u_{i}=2 \int_{\Omega} u_{i} \cdot X_{p}\left(x_{p} u_{i}\right) \\
& =2 \int_{\Omega} u_{i}^{2}+2 \int_{\Omega} x_{p} u_{i} \cdot X_{p} u_{i}
\end{aligned}
$$

and

$$
\begin{aligned}
\left\langle\left[L, y_{p}\right] u_{i}, y_{p} u_{i}\right\rangle_{L^{2}} & =-2 \int_{\Omega} Y_{p} u_{i} \cdot y_{p} u_{i}=2 \int_{\Omega} u_{i} \cdot Y_{p}\left(y_{p} u_{i}\right) \\
& =2 \int_{\Omega} u_{i}^{2}+2 \int_{\Omega} y_{p} u_{i} \cdot Y_{p} u_{i}
\end{aligned}
$$

then

$$
\left\langle\left[L, x_{p}\right] u_{i}, x_{p} u_{i}\right\rangle_{L^{2}}=\left\langle\left[L, y_{p}\right] u_{i}, y_{p} u_{i}\right\rangle_{L^{2}}=1 .
$$

On the other hand, we have

$$
\left[X_{p}, x_{p}\right] u_{i}=\left[Y_{p}, y_{p}\right] u_{i}=u_{i}
$$

and

$$
\sum_{p=1}^{n}\left\|X_{p} u_{i}\right\|_{L^{2}}^{2}+\sum_{p=1}^{n}\left\|Y_{p} u_{i}\right\|_{L^{2}}^{2}=\int_{\Omega}\left|\nabla_{\mathbb{H}^{n}} u_{i}\right|^{2}=\lambda_{i} .
$$

Thus incorporating (4.4), (4.5) and (4.6) in (4.3), we obtain (4.2). 
Remark 4.1. Inequality (4.2) improves the following inequality proved by Niu and Zhang in [25] (see Remark 5.1 in [13])

$$
\lambda_{k+1}-\lambda_{k} \leq \frac{2}{n k} \sum_{i=1}^{k} \lambda_{i}
$$

4.2. The case when $\boldsymbol{l}=\mathbf{2}$. In this subsection, we will derive the following

Theorem 4.2. We have, for each $k=1,2, \ldots$,

$\sum_{i=1}^{k}\left(\lambda_{k+1}-\lambda_{i}\right)^{2} \leq \frac{2 \sqrt{n+1}}{n}\left[\sum_{i=1}^{k}\left(\lambda_{k+1}-\lambda_{i}\right) \lambda_{i}^{\frac{1}{2}}\right]^{\frac{1}{2}}\left[\sum_{i=1}^{k}\left(\lambda_{k+1}-\lambda_{i}\right)^{2} \lambda_{i}^{\frac{1}{2}}\right]^{\frac{1}{2}}$.

Proof. The key observation here is to apply Theorem 2.1 with $A=$ $L^{2}=\left(-\Delta_{\mathbb{H}^{n}}\right)^{2}$, and as before $B_{1}=x_{1}, B_{2}=x_{2}, \cdots, B_{n}=x_{n}, B_{n+1}=$ $y_{1}, \cdots, B_{2 n}=y_{n}, T_{1}=X_{1}, \cdots, T_{n}=X_{n}, T_{n+1}=Y_{1}, \cdots, T_{2 n}=Y_{n}$ and $f(x)=g(x)=\left(\lambda_{k+1}-x\right)^{2}$. Thus we have

$$
\begin{gathered}
{\left[\sum_{p=1}^{n} \sum_{i=1}^{k}\left(\lambda_{k+1}-\lambda_{i}\right)^{2}\left(\left\langle\left[X_{p}, x_{p}\right] u_{i}, u_{i}\right\rangle_{L^{2}}+\left\langle\left[Y_{p}, y_{p}\right] u_{i}, u_{i}\right\rangle_{L^{2}}\right)\right]^{2}} \\
\leq 4\left[\sum_{p=1}^{n} \sum_{i=1}^{k}\left(\lambda_{k+1}-\lambda_{i}\right)^{2}\left(\left\langle\left[L^{2}, x_{p}\right] u_{i}, x_{p} u_{i}\right\rangle_{L^{2}}+\left\langle\left[L^{2}, y_{p}\right] u_{i}, y_{p} u_{i}\right\rangle_{L^{2}}\right)\right] \\
\times\left[\sum_{p=1}^{n} \sum_{i=1}^{k}\left(\lambda_{k+1}-\lambda_{i}\right)\left(\left\|X_{p} u_{i}\right\|_{L^{2}}^{2}+\left\|Y_{p} u_{i}\right\|_{L^{2}}^{2}\right)\right]
\end{gathered}
$$

but

$$
\begin{aligned}
\sum_{p=1}^{n}\left\|X_{p} u_{i}\right\|_{L^{2}}^{2}+\sum_{p=1}^{n}\left\|Y_{p} u_{i}\right\|_{L^{2}}^{2} & =\int_{\Omega}\left|\nabla_{\mathbb{H}^{n}} u_{i}\right|^{2}=\int_{\Omega} L u_{i} \cdot u_{i} \\
& \leq\left(\int_{\Omega} u_{i}^{2}\right)^{\frac{1}{2}}\left(\int_{\Omega}\left(L u_{i}\right)^{2}\right)^{\frac{1}{2}}=\lambda_{i}^{\frac{1}{2}},
\end{aligned}
$$

thus

$$
\sum_{p=1}^{n} \sum_{i=1}^{k}\left(\lambda_{k+1}-\lambda_{i}\right)\left(\left\|X_{p} u_{i}\right\|_{L^{2}}^{2}+\left\|Y_{p} u_{i}\right\|_{L^{2}}^{2}\right)=\sum_{i=1}^{k}\left(\lambda_{k+1}-\lambda_{i}\right) \lambda_{i}^{\frac{1}{2}} .
$$

Using (4.5), we get

$$
\left\langle\left[X_{p}, x_{p}\right] u_{i}, u_{i}\right\rangle_{L^{2}}=\left\langle\left[Y_{p}, y_{p}\right] u_{i}, u_{i}\right\rangle_{L^{2}}=1
$$


Thus,

$$
\begin{gathered}
{\left[\sum_{p=1}^{n} \sum_{i=1}^{k}\left(\lambda_{k+1}-\lambda_{i}\right)^{2}\left(\left\langle\left[X_{p}, x_{p}\right] u_{i}, u_{i}\right\rangle_{L^{2}}+\left\langle\left[Y_{p}, y_{p}\right] u_{i}, u_{i}\right\rangle_{L^{2}}\right)\right]^{2}} \\
=4 n^{2}\left[\sum_{i=1}^{k}\left(\lambda_{k+1}-\lambda_{i}\right)^{2}\right]^{2}
\end{gathered}
$$

On the other hand

$$
\begin{aligned}
{\left[L^{2}, x_{p}\right] u_{i}=L^{2}\left(x_{p} u_{i}\right)-x_{p} L^{2} u_{i} } & =L\left(x_{p} L u_{i}-2 X_{p} u_{i}\right)-x_{p} L^{2} u_{i} \\
& =-2 X_{p} L u_{i}-2 L\left(X_{p} u_{i}\right)
\end{aligned}
$$

and the same identity holds with $y_{p}$ and $Y_{p}$.

We infer, using identities (4.5) and (4.13)

$$
\begin{aligned}
\left\langle\left[L^{2}, x_{p}\right] u_{i}, x_{p} u_{i}\right\rangle_{L^{2}}=-2 \int_{\Omega} X_{p} L u_{i} \cdot x_{p} u_{i}-2 \int_{\Omega} L\left(X_{p} u_{i}\right) \cdot x_{p} u_{i} \\
=-2 \int_{\Omega} X_{p} L u_{i} \cdot x_{p} u_{i}-2 \int_{\Omega} X_{p} u_{i} \cdot x_{p} L u_{i}+4 \int_{\Omega} X_{p} u_{i} \cdot X_{p} u_{i} \\
=2 \int_{\Omega} L u_{i} \cdot X_{p}\left(x_{p} u_{i}\right)-2 \int_{\Omega} x_{p} X_{p} u_{i} \cdot L u_{i}-4 \int_{\Omega} X_{p}^{2} u_{i} \cdot u_{i} \\
=2 \int_{\Omega} L u_{i} \cdot u_{i}-4 \int_{\Omega} X_{p}^{2} u_{i} \cdot u_{i} .
\end{aligned}
$$

Similarly, we have

$$
\left\langle\left[L^{2}, y_{p}\right] u_{i}, y_{p} u_{i}\right\rangle_{L^{2}}=2 \int_{\Omega} L u_{i} \cdot u_{i}-4 \int_{\Omega} Y_{p}^{2} u_{i} \cdot u_{i}
$$

Since

$$
\begin{aligned}
-\sum_{p=1}^{n} \int_{\Omega} X_{p}^{2} u_{i} \cdot u_{i}-\sum_{p=1}^{n} \int_{\Omega} Y_{p}^{2} u_{i} \cdot u_{i} & =\sum_{p=1}^{n}\left\|X_{p} u_{i}\right\|_{L^{2}}^{2}+\sum_{p=1}^{n}\left\|Y_{p} u_{i}\right\|_{L^{2}}^{2} \\
& =\int_{\Omega} L u_{i} \cdot u_{i},
\end{aligned}
$$


we have

$$
\begin{aligned}
{\left[\sum _ { p = 1 } ^ { n } \sum _ { i = 1 } ^ { k } \left(\lambda_{k+1}\right.\right.} & \left.\left.-\lambda_{i}\right)^{2}\left(\left\langle\left[L^{2}, x_{p}\right] u_{i}, x_{p} u_{i}\right\rangle_{L^{2}}+\left\langle\left[L^{2}, y_{p}\right] u_{i}, y_{p} u_{i}\right\rangle_{L^{2}}\right)\right] \\
& =4(n+1) \sum_{i=1}^{k}\left(\lambda_{k+1}-\lambda_{i}\right)^{2} \int_{\Omega} L u_{i} \cdot u_{i} \\
& \leq 4(n+1) \sum_{i=1}^{k}\left(\lambda_{k+1}-\lambda_{i}\right)^{2}\left(\int_{\Omega} u_{i}^{2}\right)^{\frac{1}{2}}\left(\int_{\Omega}\left(L u_{i}\right)^{2}\right)^{\frac{1}{2}} \\
& =4(n+1) \sum_{i=1}^{k}\left(\lambda_{k+1}-\lambda_{i}\right)^{2} \lambda_{i}^{\frac{1}{2}} .
\end{aligned}
$$

Incorporating (4.10), (4.12) and (4.16) in (4.8), we get the result.

We can easily obtain from inequality (4.7) of Theorem 4.2 an inequality of Yang-type.

Corollary 4.1. We have, for each $k \geq 1$,

$$
\sum_{i=1}^{k}\left(\lambda_{k+1}-\lambda_{i}\right)^{2} \leq \frac{4(n+1)}{n^{2}} \sum_{i=1}^{k}\left(\lambda_{k+1}-\lambda_{i}\right) \lambda_{i}
$$

Proof. Inequality (4.7) is equivalent to

$$
\left[\sum_{i=1}^{k}\left(\lambda_{k+1}-\lambda_{i}\right)^{2}\right]^{2} \leq \frac{4(n+1)}{n^{2}}\left[\sum_{i=1}^{k}\left(\lambda_{k+1}-\lambda_{i}\right) \lambda_{i}^{\frac{1}{2}}\right]\left[\sum_{i=1}^{k}\left(\lambda_{k+1}-\lambda_{i}\right)^{2} \lambda_{i}^{\frac{1}{2}}\right] .
$$

Now applying Lemma 3.2 with $A_{i}=\lambda_{k+1}-\lambda_{i}$ and $B_{i}=C_{i}=\lambda_{i}^{\frac{1}{2}}$, we obtain inequality (4.17).

Remark 4.2. Inequality (4.7) is sharper than the following one found by Niu and Zhang [25]

$$
\lambda_{k+1}-\lambda_{k} \leq \frac{4(n+1)}{n^{2} k^{2}}\left(\sum_{i=1}^{k} \lambda_{i}^{\frac{1}{2}}\right)^{2} .
$$

Proof. We infer from inequality (4.7) and the Chebyshev inequality

$$
\begin{gathered}
{\left[\sum_{i=1}^{k}\left(\lambda_{k+1}-\lambda_{i}\right)^{2}\right]^{2}} \\
\leq \frac{4(n+1)}{n^{2} k^{2}}\left[\sum_{i=1}^{k}\left(\lambda_{k+1}-\lambda_{i}\right)\right]\left[\sum_{i=1}^{k}\left(\lambda_{k+1}-\lambda_{i}\right)^{2}\right]\left[\sum_{i=1}^{k} \lambda^{\frac{1}{2}}\right]^{2},
\end{gathered}
$$

or equivalently

$$
\sum_{i=1}^{k}\left(\lambda_{k+1}-\lambda_{i}\right)^{2} \leq \frac{4(n+1)}{n^{2} k^{2}}\left[\sum_{i=1}^{k}\left(\lambda_{k+1}-\lambda_{i}\right)\right]\left[\sum_{i=1}^{k} \lambda^{\frac{1}{2}}\right]^{2}
$$


Thus

$$
\sum_{i=1}^{k}\left(\lambda_{k+1}-\lambda_{i}\right)\left[\left(\lambda_{k+1}-\lambda_{i}\right)-\frac{4(n+1)}{n^{2} k^{2}}\left[\sum_{i=1}^{k} \lambda_{i}^{\frac{1}{2}}\right]^{2}\right] \leq 0 .
$$

Hence, since $\lambda_{i} \leq \lambda_{k}$, for all $i \leq k$, we can easily deduce the inequality of Niu and Zhang from (4.18).

4.3. The case when $\boldsymbol{l} \geq 3$. We are now concerned with the problem (4.1) for any $l \geq 3$. The result depends on the parity of $l$. In fact, we prove the following

Theorem 4.3. For any odd $l \geq 3$, we have

$$
\begin{aligned}
& \sum_{i=1}^{k}\left(\lambda_{k+1}-\lambda_{i}\right)^{2} \leq \frac{1}{n}\left[\sum_{i=1}^{k}\left(\lambda_{k+1}-\lambda_{i}\right) \lambda_{i}^{\frac{1}{l}}\right]^{\frac{1}{2}} \times \\
& \quad\left\{\sum_{i=1}^{k}\left(\lambda_{k+1}-\lambda_{i}\right)^{2}\left[(2 l(n+l-1)) \lambda_{i}^{\frac{l-1}{l}}+c_{1}(n, l)\left(\lambda_{i}+\lambda_{i}^{\frac{l-2}{l}}\right)\right]\right\}^{\frac{1}{2}}
\end{aligned}
$$

and for any even $l \geq 4$, we have

$$
\begin{aligned}
\sum_{i=1}^{k}\left(\lambda_{k+1}-\lambda_{i}\right)^{2} \leq \frac{1}{n}\left[\sum_{i=1}^{k}\left(\lambda_{k+1}-\lambda_{i}\right) \lambda_{i}^{\frac{1}{l}}\right]^{\frac{1}{2}} \times \\
\left.\left\{\sum_{i=1}^{k}\left(\lambda_{k+1}-\lambda_{i}\right)^{2}\left[(2 \ln +4(l-1)) \lambda_{i}^{\frac{l-1}{l}}+c_{2}(n, l) \lambda_{i}^{\frac{l-1}{l}}\right)\right]\right\}^{\frac{1}{2}}
\end{aligned}
$$

where $c_{1}(n, l)$ and $c_{2}(n, l)$ are two constants depending on $n$ and $l$.

Proof. If we apply inequality (2.2) with $A=L^{l}=\left(-\Delta_{\mathbb{H} n}\right)^{l}, B_{1}=$ $x_{1}, \ldots, B_{n}=x_{n}, B_{n+1}=y_{1}, \ldots, B_{2 n}=y_{n}, T_{1}=X_{1}, \ldots, T_{n}=X_{n}, T_{n+1}=$ $Y_{1}, \ldots, T_{2 n}=Y_{n}$ and $f(x)=g(x)=\left(\lambda_{k+1}-x\right)^{2}$, then we obtain

$$
\begin{gathered}
{\left[\sum_{i=1}^{k} \sum_{p=1}^{n}\left(\lambda_{k+1}-\lambda_{i}\right)^{2}\left(\left\langle\left[X_{p}, x_{p}\right] u_{i}, u_{i}\right\rangle_{L^{2}}+\left\langle\left[Y_{p}, y_{p}\right] u_{i}, u_{i}\right\rangle_{L^{2}}\right)\right]^{2}} \\
\leq 4\left[\sum_{i=1}^{k} \sum_{p=1}^{n}\left(\lambda_{k+1}-\lambda_{i}\right)^{2}\left(\left\langle\left[L^{l}, x_{p}\right] u_{i}, x_{p} u_{i}\right\rangle_{L^{2}}+\left\langle\left[L^{l}, y_{p}\right] u_{i}, y_{p} u_{i}\right\rangle_{L^{2}}\right)\right] \times \\
{\left[\sum_{i=1}^{k} \sum_{p=1}^{n}\left(\lambda_{k+1}-\lambda_{i}\right)\left(\left\|X_{p} u_{i}\right\|_{L^{2}}^{2}+\left\|Y_{p} u_{i}\right\|_{L^{2}}^{2}\right)\right]}
\end{gathered}
$$

And as before, we have

$$
\left\langle\left[X_{p}, x_{p}\right] u_{i}, u_{i}\right\rangle_{L^{2}}=\left\langle\left[Y_{p}, y_{p}\right] u_{i}, u_{i}\right\rangle_{L^{2}}=1 .
$$


On the other hand, to calculate $\sum_{p=1}^{n}\left(\left\|X_{p} u_{i}\right\|_{L^{2}}^{2}+\left\|Y_{p} u_{i}\right\|_{L^{2}}^{2}\right)$, we need the following result obtained by Niu and Zhang (see Lemma 2.3 in [25]) inspired by that of Chen and Qian [7] for the Laplacian:

Lemma 4.1. For any $d \geq 1$, we have

$$
\left(\int_{\Omega}\left|\nabla_{\mathbb{H}^{n}}^{d} u_{i}\right|^{2}\right)^{\frac{1}{d}} \leq\left(\int_{\Omega}\left|\nabla_{\mathbb{H}^{n}}^{d+1} u_{i}\right|^{2}\right)^{\frac{1}{d+1}}
$$

where $\nabla^{d}= \begin{cases}L^{\frac{d}{2}} & \text { if d is even, } \\ \nabla_{\mathbb{H}^{n}} L^{\frac{d-1}{2}} & \text { if } d \text { is odd. }\end{cases}$

And as a consequence (see Corollary 2.1 in [25]), we can easily obtain, for any $d \geq 1$

$$
\left(\int_{\Omega}\left|\nabla_{\mathbb{H}^{n}} u_{i}\right|^{2}\right) \leq\left(\int_{\Omega}\left|\nabla_{\mathbb{H}^{n}}^{d} u_{i}\right|^{2}\right)^{\frac{1}{d}} .
$$

Therefore we have

$$
\sum_{p=1}^{n}\left(\left\|X_{p} u_{i}\right\|_{L^{2}}^{2}+\left\|Y_{p} u_{i}\right\|_{L^{2}}^{2}\right)=\int_{\Omega} L u_{i} \cdot u_{i} \leq\left(\int_{\Omega} L^{l} u_{i} \cdot u_{i}\right)^{\frac{1}{l}}=\lambda_{i}^{\frac{1}{l}} .
$$

Now we have to calculate

$$
\sum_{p=1}^{n}\left(\left\langle\left[L^{l}, x_{p}\right] u_{i}, x_{p} u_{i}\right\rangle_{L^{2}}+\left\langle\left[L^{l}, y_{p}\right] u_{i}, y_{p} u_{i}\right\rangle_{L^{2}}\right) .
$$

For this purpose, we use the following lemma also obtained by Niu and Zhang in [25]

Lemma 4.2. For any positive integer $d, 1 \leq d \leq l$, we have

$$
L^{d}\left(x_{p} u_{i}\right)=x_{p} L^{d} u_{i}-2 \sum_{q=1}^{d} L^{d-q} X_{p} L^{q-1} u_{i},
$$

$i=1, \ldots, k, p=1, \ldots, n$. This is also true for $y_{p}$ and $Y_{p}$.

We infer, using Lemma 4.2 ,

$$
\left[L^{l}, x_{p}\right] u_{i}=L^{l}\left(x_{p} u_{i}\right)-x_{p} L^{l} u_{i}=-2 \sum_{q=1}^{l} L^{l-q} X_{p} L^{q-1} u_{i} .
$$

Therefore

$$
\begin{aligned}
\left\langle\left[L^{l}, x_{p}\right] u_{i}, x_{p} u_{i}\right\rangle_{L^{2}} & =-2 \sum_{q=1}^{l} \int_{\Omega} L^{l-q} X_{p} L^{q-1} u_{i} \cdot x_{p} u_{i} \\
& =-2 \sum_{q=1}^{l} \int_{\Omega} X_{p} L^{q-1} u_{i} \cdot L^{l-q}\left(x_{p} u_{i}\right) .
\end{aligned}
$$


The same identities hold with $y_{p}$ and $Y_{p}$.

Hence we obtain

$$
\begin{gathered}
\sum_{p=1}^{n}\left(\left\langle\left[L^{l}, x_{p}\right] u_{i}, x_{p} u_{i}\right\rangle_{L^{2}}+\left\langle\left[L^{l}, y_{p}\right] u_{i}, y_{p} u_{i}\right\rangle_{L^{2}}\right) \\
=-2 \sum_{p=1}^{n} \sum_{q=1}^{l}\left(\int_{\Omega} X_{p} L^{q-1} u_{i} . L^{l-q}\left(x_{p} u_{i}\right)+\int_{\Omega} Y_{p} L^{q-1} u_{i} \cdot L^{l-q}\left(y_{p} u_{i}\right)\right) .
\end{gathered}
$$

Applying Lemma 4.2 once again to (4.26), we obtain

$$
\begin{gathered}
\sum_{p=1}^{n}\left(\left\langle\left[L^{l}, x_{p}\right] u_{i}, x_{p} u_{i}\right\rangle_{L^{2}}+\left\langle\left[L^{l}, y_{p}\right] u_{i}, y_{p} u_{i}\right\rangle_{L^{2}}\right) \\
=-2 \sum_{p=1}^{n} \sum_{q=1}^{l} \int_{\Omega} x_{p} L^{l-q} u_{i} . X_{p} L^{q-1} u_{i}+4 \sum_{p=1}^{n} \sum_{q=1}^{l-1} \int_{\Omega} X_{p} L^{l-q-1} u_{i} . X_{p} L^{q-1} u_{i} \\
+4 \sum_{p=1}^{n} \sum_{q=1}^{l-2} \sum_{r=1}^{l-q-1} \int_{\Omega} L^{l-q-r} X_{p} L^{r-1} u_{i} \cdot X_{p} L^{q-1} u_{i} \\
-2 \sum_{p=1}^{n} \sum_{q=1}^{l} \int_{\Omega} y_{p} L^{l-q} u_{i} . Y_{p} L^{q-1} u_{i}+4 \sum_{p=1}^{n} \sum_{q=1}^{l-1} \int_{\Omega} Y_{p} L^{l-q-1} u_{i} \cdot Y_{p} L^{q-1} u_{i} \\
+4 \sum_{p=1}^{n} \sum_{q=1}^{l-2} \sum_{r=1}^{l-q-1} \int_{\Omega} L^{l-q-r} Y_{p} L^{r-1} u_{i} . Y_{p} L^{q-1} u_{i} . \quad(4.27)
\end{gathered}
$$

As in the proof of Theorem 5.1 in 25] (see the calculation of the terms $I_{2}$ and $I_{2}^{\prime}$ ), we can easily obtain, for any odd $l \geq 3$,

$$
\begin{aligned}
& \sum_{p=1}^{n}\left(\left\langle\left[L^{l}, x_{p}\right] u_{i}, x_{p} u_{i}\right\rangle_{L^{2}}+\left\langle\left[L^{l}, y_{p}\right] u_{i}, y_{p} u_{i}\right\rangle_{L^{2}}\right) \\
\leq & (2 l(n+l-1)) \lambda_{i}^{\frac{l-1}{l}}+c_{1}(n, l)\left(\lambda_{i}+\lambda_{i}^{\frac{l-2}{l}}\right)
\end{aligned}
$$

and for any even $l \geq 4$,

$$
\begin{aligned}
& \sum_{p=1}^{n}\left(\left\langle\left[L^{l}, x_{p}\right] u_{i}, x_{p} u_{i}\right\rangle_{L^{2}}+\left\langle\left[L^{l}, y_{p}\right] u_{i}, y_{p} u_{i}\right\rangle_{L^{2}}\right) \\
\leq & {\left[(2 \ln +4(l-1))+c_{2}(n, l)\right] \lambda_{i}^{\frac{l-1}{l}} }
\end{aligned}
$$

where $c_{1}(n, 3)=4$,

$c_{1}(n, l)=2 \sum_{q=1}^{l-2} \sum_{r=1}^{l-q-1}\left\{\sum_{\substack{s=1 \\ \text { sodd }}}^{l-q-r} \frac{2^{s} n C_{l-q-r}^{s}}{(2 n-1)^{\frac{s+1}{2}}}+\sum_{\substack{s=2 \\ \text { seven }}}^{l-q-r} \frac{2^{s} C_{l-q-r}^{s}}{(2 n-1)^{\frac{s}{2}}}\right\}$ for any odd 
$l \geq 5$ and $c_{2}(n, l)=4 \sum_{q=1}^{l-2} \sum_{r=1}^{l-q-1}\left\{\sum_{\substack{s=1 \\ \text { sodd }}}^{l-q-r} \frac{2^{s} n C_{l-q-r}^{s}}{(2 n-1)^{\frac{s+1}{2}}}+\sum_{\substack{s=0 \\ \text { seven }}}^{l-q-r} \frac{2^{s} C_{l-q-r}^{s}}{(2 n-1)^{\frac{s}{2}}}\right\}$. Incorporating (4.22), (4.25) and (4.28) in (4.21), we obtain (4.19). Similarly, to obtain (4.20), we incorporate (4.22), (4.25) and (4.29) in (4.21).

Remark 4.3. The inequality $\sqrt{4.19}$ is not homogeneous in the eigenvalues $\lambda_{i}$ (i.e. it is not invariant under the change $L \rightarrow a L, \lambda_{i} \rightarrow a^{l} \lambda_{i}$ for $a>0)$. Therefore, using inequality (4.19) for $a^{l} L^{l}$, we obtain that for any $a>0$,

$$
\begin{aligned}
& \sum_{i=1}^{k}\left(\lambda_{k+1}-\lambda_{i}\right)^{2} \leq \frac{1}{n}\left[\sum_{i=1}^{k}\left(\lambda_{k+1}-\lambda_{i}\right) \lambda_{i}^{\frac{1}{l}}\right]^{\frac{1}{2}} \times \\
& \quad\left\{\sum_{i=1}^{k}\left(\lambda_{k+1}-\lambda_{i}\right)^{2}\left[2 l(n+l-1) \lambda_{i}^{\frac{l-1}{l}}+c_{1}(n, l)\left(a \lambda_{i}+\frac{1}{a} \lambda_{i}^{\frac{l-2}{l}}\right)\right]\right\}^{\frac{1}{2}},
\end{aligned}
$$

for any odd $l \geq 3$.

Optimising with respect to a, we find the following improvement of the inequality 4.19)

$$
\begin{aligned}
\sum_{i=1}^{k}\left(\lambda_{k+1}-\lambda_{i}\right)^{2} \leq \frac{1}{n}(2 l(n+l-1)+ & \left.c_{1}(n, l)\right)^{\frac{1}{2}}\left[\sum_{i=1}^{k}\left(\lambda_{k+1}-\lambda_{i}\right) \lambda_{i}^{\frac{1}{l}}\right]^{\frac{1}{2}} \times \\
& {\left[\sum_{i=1}^{k}\left(\lambda_{k+1}-\lambda_{i}\right)^{2} \lambda_{i}^{\frac{l-1}{l}}\right]^{\frac{1}{2}} }
\end{aligned}
$$

which is homogeneous on the eigenvalues $\lambda_{i}$.

As for the case when $l=2$, we can deduce inequalities of Yang-type for $l \geq 3$.

Corollary 4.2. We have, for any odd $l \geq 3$,

$$
\begin{gathered}
\sum_{i=1}^{k}\left(\lambda_{k+1}-\lambda_{i}\right)^{2} \\
\leq \frac{1}{n^{2}} \sum_{i=1}^{k}\left(\lambda_{k+1}-\lambda_{i}\right)\left[(2 l(n+l-1)) \lambda_{i}+c_{1}(n, l)\left(\lambda_{i}^{\frac{l+1}{l}}+\lambda_{i}^{\frac{l-1}{l}}\right)\right]
\end{gathered}
$$

and for any even $l \geq 4$

$$
\sum_{i=1}^{k}\left(\lambda_{k+1}-\lambda_{i}\right)^{2} \leq \frac{2 \ln +4(l-1)+c_{2}(n, l)}{n^{2}} \sum_{i=1}^{k}\left(\lambda_{k+1}-\lambda_{i}\right) \lambda_{i} .
$$

where $c_{1}(n, l)$ and $c_{2}(n, l)$ are explicit constants depending only on $n$ and $l$. 
Proof. Applying Lemma 3.2 with $A_{i}=\lambda_{k+1}-\lambda_{i}, B_{i}=2 l(n+l-$ 1) $\lambda_{i}^{\frac{l-1}{l}}+c_{1}(n, l)\left(\lambda_{i}+\lambda_{i}^{\frac{l-2}{l}}\right)$ and $C_{i}=\lambda_{i}^{\frac{1}{l}}$, we obtain

$$
\begin{gathered}
{\left[\sum_{i=1}^{k}\left(\lambda_{k+1}-\lambda_{i}\right) \lambda_{i}^{\frac{1}{l}}\right]\left\{\sum _ { i = 1 } ^ { k } ( \lambda _ { k + 1 } - \lambda _ { i } ) ^ { 2 } \left[2 l(n+l-1) \lambda_{i}^{\frac{l-1}{l}}\right.\right.} \\
\left.\left.+c_{1}(n, l)\left(\lambda_{i}+\lambda_{i}^{\frac{l-2}{l}}\right)\right]\right\} \leq \sum_{i=1}^{k}\left(\lambda_{k+1}-\lambda_{i}\right)^{2} \times \\
\quad \sum_{i=1}^{k}\left(\lambda_{k+1}-\lambda_{i}\right) \lambda_{i}^{\frac{1}{l}}\left[2 l(n+l-1) \lambda_{i}^{\frac{l-1}{l}}+c_{1}(n, l)\left(\lambda_{i}+\lambda_{i}^{\frac{l-2}{l}}\right)\right] \\
=\sum_{i=1}^{k}\left(\lambda_{k+1}-\lambda_{i}\right)^{2} \sum_{i=1}^{k}\left(\lambda_{k+1}-\lambda_{i}\right)\left[2 l(n+l-1) \lambda_{i}+c_{1}(n, l)\left(\lambda_{i}^{\frac{l+1}{l}}+\lambda_{i}^{\frac{l-1}{l}}\right)\right]
\end{gathered}
$$

Inequality (4.32) can be deduced from (4.19) and (4.34), for any odd $l \geq 3$.

We proceed in the same way to obtain (4.33), i.e. applying Lemma 3.2 but with $A_{i}=\lambda_{k+1}-\lambda_{i}, B_{i}=\left(2 \ln +4(l-1)+c_{2}(n, l)\right) \lambda_{i}^{\frac{l-1}{l}}$ and $C_{i}=\lambda_{i}^{\frac{1}{l}}$

Remark 4.4. Inequalities (4.19) and (4.20) are sharper than the following inequalities, proved by Niu and Zhang (25]),

$\lambda_{k+1}-\lambda_{k} \leq \frac{\sum_{i=1}^{k} \lambda_{i}^{\frac{1}{l}}}{n^{2} k^{2}}\left[(2 l(n+l-1)) \sum_{i=1}^{k} \lambda_{i}^{\frac{l-1}{l}}+c_{1}(n, l) \sum_{i=1}^{k}\left(\lambda_{i}+\lambda_{i}^{\frac{l-2}{l}}\right)\right]$

if $l \geq 3$ is odd and

$$
\lambda_{k+1}-\lambda_{k} \leq \frac{\sum_{i=1}^{k} \lambda_{i}^{\frac{1}{l}}}{n^{2} k^{2}}\left[(2 \ln +4(l-1)) \sum_{i=1}^{k} \lambda_{i}^{\frac{l-1}{l}}+c_{2}(n, l) \sum_{i=1}^{k} \lambda_{i}^{\frac{l-1}{l}}\right],
$$

if $l \geq 4$ is even,

$c_{1}(n, l)$ and $c_{2}(n, l)$ are as in the proof of Theorem 4.3 .

Proof. By the Chebyshev inequality, we infer from (4.19), for any odd $l \geq 3$

$$
\begin{array}{r}
{\left[\sum_{i=1}^{k}\left(\lambda_{k+1}-\lambda_{i}\right)^{2}\right]^{2} \leq \frac{1}{n^{2} k^{2}}\left[\sum_{i=1}^{k}\left(\lambda_{k+1}-\lambda_{i}\right)\right]\left[\sum_{i=1}^{k}\left(\lambda_{k+1}-\lambda_{i}\right)^{2}\right] \times} \\
{\left[\sum_{i=1}^{k} \lambda_{i}^{\frac{1}{l}}\right]\left[2 l(n+l-1) \sum_{i=1}^{k} \lambda_{i}^{\frac{l-1}{l}}+c_{1}(n, l) \sum_{i=1}^{k}\left(\lambda_{i}+\lambda_{i}^{\frac{l-2}{l}}\right)\right]}
\end{array}
$$


or equivalently

$$
\begin{gathered}
\sum_{i=1}^{k}\left(\lambda_{k+1}-\lambda_{i}\right)^{2} \leq \frac{1}{n^{2} k^{2}}\left[\sum_{i=1}^{k}\left(\lambda_{k+1}-\lambda_{i}\right)\right]\left[\sum_{i=1}^{k} \lambda_{i}^{\frac{1}{l}}\right] \times \\
{\left[2 l(n+l-1) \sum_{i=1}^{k} \lambda_{i}^{\frac{l-1}{l}}+c_{1}(n, l) \sum_{i=1}^{k}\left(\lambda_{i}+\lambda_{i}^{\frac{l-2}{l}}\right)\right] .}
\end{gathered}
$$

Thus

$$
\begin{aligned}
\sum_{i=1}^{k}\left(\lambda_{k+1}-\lambda_{i}\right) & {\left[\lambda_{k+1}-\lambda_{i}-\frac{1}{n^{2} k^{2}}\left(\sum_{i=1}^{k} \lambda_{i}^{\frac{1}{l}}\right) \times\right.} \\
& \left.\left(2 l(n+l-1) \sum_{i=1}^{k} \lambda_{i}^{\frac{l-1}{l}}+c_{1}(n, l) \sum_{i=1}^{k}\left(\lambda_{i}+\lambda_{i}^{\frac{l-2}{l}}\right)\right)\right] \leq 0
\end{aligned}
$$

which implies (4.35), since $\lambda_{i} \leq \lambda_{k}$ for $i \leq k$.

Similarly, we prove that inequality (4.20) is sharper than (4.36).

Acknowledgments. We thank the referee for the suggestions which allowed us to improve the first version of the paper.

This work was partially supported by the ANR (Agence Nationale de la Recherche) through FOG project(ANR-07-BLAN-0251-01).

\section{REFERENCES}

[1] M. S. Ashbaugh. Isoperimetric and universal inequalities for eigenvalues. In Spectral theory and geometry (Edinburgh, 1998), volume 273 of London Math. Soc. Lecture Note Ser., pages 95-139. Cambridge Univ. Press, Cambridge, 1999.

[2] M. S. Ashbaugh. The universal eigenvalue bounds of Payne-Pólya-Weinberger, Hile-Protter, and H. C. Yang. Proc. Indian Acad. Sci. Math. Sci., 112(1):3-30, 2002. Spectral and inverse spectral theory (Goa, 2000).

[3] M. S. Ashbaugh and L. Hermi. Universal inequalities for higher-order elliptic operators, preprint 2003.

[4] M.S. Ashbaugh and L. Hermi. A unified approach to universal inequalities for eigenvalues of elliptic operators. Pacific J. Math., 217(2):201-219, 2004.

[5] M.S. Ashbaugh and L. Hermi. On harrell-stubbe type inequalities for the discrete spectrum of a self-adjoint operator. arXiv: 0712.4396v1 [math.SP], 28th of december 2007.

[6] Z. Chen and C. Qian. Hile-Yeh estimates of eigenvalues of polyharmonic operators. Acta Math. Sinica (N.S.), 9(4):432-437, 1993. A Chinese summary appears in Acta Math. Sinica 37 (1994), no. 5, 719.

[7] Z. C. Chen and C. L. Qian. Estimates for discrete spectrum of the laplacian operator with any order. J. China Univ. Sci. Tech., 20:259-266, 1990.

[8] Z. C. Chen and C. L. Qian. On the difference of consecutive eigenvalues of uniformly elliptic operators of higher orders. Chinese Ann. Math. Ser. B, 14(4):435-442, 1993. A Chinese summary appears in Chinese Ann. Math. Ser. A 14 (1993), no. 6, 740 . 
[9] Z. C. Chen and C. L. Qian. On the upper bound of eigenvalues for elliptic equations with higher orders. J. Math. Anal. Appl., 186(3):821-834, 1994.

[10] Q.-M. Cheng, T. Ichikawa, and S. Mametsuka. Inequalities for eigenvalues of laplacian with any order, preprint 2009.

[11] Q.-M. Cheng and H.C. Yang. Inequalities for eigenvalues of a clamped plate problem. Trans. Amer. Math. Soc., 358(6):2625-2635 (electronic), 2006.

[12] Q.M. Cheng and H.C. Yang. Estimates on eigenvalues of laplacian. Math. Annal., 331:445-460, 2005.

[13] A. El Soufi, E.M. Harrell II, and S. Ilias. Universal inequalities for the eigenvalues of laplace and schrodinger operators on submanifolds. arXiv, to appear in Transactions of the AMS.

[14] E.M. Harrell, II. General bounds for the eigenvalues of Schrödinger operators. In Maximum principles and eigenvalue problems in partial differential equations (Knoxville, TN, 1987), volume 175 of Pitman Res. Notes Math. Ser., pages 146-166. Longman Sci. Tech., Harlow, 1988.

[15] E.M. Harrell, II and P.L. Michel. Commutator bounds for eigenvalues of some differential operators. In Evolution equations (Baton Rouge, LA, 1992), volume 168 of Lecture Notes in Pure and Appl. Math., pages 235-244. Dekker, New York, 1995.

[16] E.M. Harrell, II and P.L. Michel. Erratum to: "Commutator bounds for eigenvalues, with applications to spectral geometry" [Comm. Partial Differential Equations 19 (1994), no. 11-12; 2037-2055; MR1301181 (95i:58182)]. Comm. Partial Differential Equations, 20(7-8):1453, 1995.

[17] Evans M. Harrell, II. Some geometric bounds on eigenvalue gaps. Comm. Partial Differential Equations, 18(1-2):179-198, 1993.

[18] E.M. Harrell II and J. Stubbe. Universal bounds and semiclassical estimates for eigenvalues of abstract schrödinger operators. To appear in Math. Annal.

[19] E.M. Harrell II and J. Stubbe. On trace identities and universal eigenvalue estimates for some partial differential operators. Trans. Amer. Math. Soc., 349(5):1797-1809, 1997.

[20] G. N. Hile and M. H. Protter. Inequalities for eigenvalues of the Laplacian. Indiana Univ. Math. J., 29(4):523-538, 1980.

[21] G. N. Hile and R. Z. Yeh. Inequalities for eigenvalues of the biharmonic operator. Pacific J. Math., 112(1):115-133, 1984.

[22] S. M. Hook. Domain-independent upper bounds for eigenvalues of elliptic operators. Trans. Amer. Math. Soc., 318(2):615-642, 1990.

[23] S. M. Hook. Inequalities for eigenvalues of self-adjoint operators. Trans. Amer. Math. Soc., 318:237-259, 1990.

[24] M. Levitin and L. Parnovski. Commutators, spectral trace identities, and universal estimates for eigenvalues. J. Funct. Anal., 192(2):425-445, 2002.

[25] P. Niu and H. Zhang. Payne-Polya-Weinberger type inequalities for eigenvalues of nonelliptic operators. Pacific J. Math., 208(2):325-345, 2003.

[26] L. E. Payne, G. Pólya, and H. F. Weinberger. On the ratio of consecutive eigenvalues. J. Math. Phys., 35:289-298, 1956.

[27] C. J. Thompson. On the ratio of consecutive eigenvalues in $N$-dimensions. Studies in Appl. Math., 48:281-283, 1969.

[28] F. Wu and L. Cao. Estimates for eigenvalues of Laplacian operator with any order. Sci. China Ser. A, 50(8):1078-1086, 2007.

[29] H.C. Yang. An estimate of the difference between consecutive eigenvalues. preprint IC/91/60 of the Intl. Center for Theoretical Physics, Trieste, 1991(revised preprint, Academia Sinica, 1995). 
S. Ilias, O. Makhoul: Université François rabelais de Tours, Laboratoire de Mathématiques et Physique Théorique, UMR-CNRS 6083, Parc de Grandmont, 37200 Tours, France

E-mail address: ilias@univ-tours.fr, ola.makhoul@lmpt.univ-tours.fr 